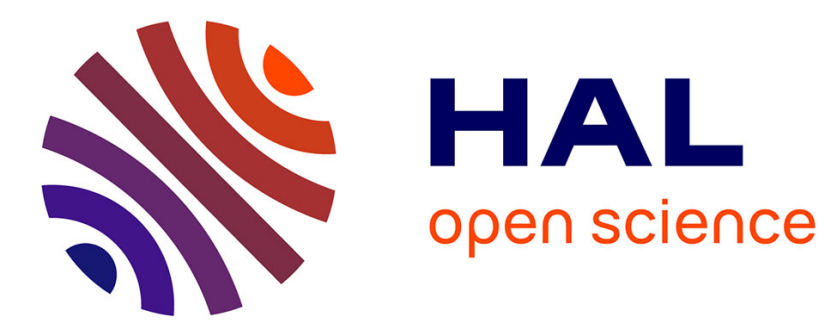

\title{
Pressure-dependent threshold in a granular flow: numerical modelling and experimental validation
}

Laurent Chupin, Thierry Dubois, Minh Phan, Olivier Roche

\section{To cite this version:}

Laurent Chupin, Thierry Dubois, Minh Phan, Olivier Roche. Pressure-dependent threshold in a granular flow: numerical modelling and experimental validation. Journal of Non-Newtonian Fluid Mechanics, 2021, 10.1016/j.jnnfm.2021.104529 . hal-02562000v2

\section{HAL Id: hal-02562000 \\ https://hal.science/hal-02562000v2}

Submitted on 12 Nov 2021

HAL is a multi-disciplinary open access archive for the deposit and dissemination of scientific research documents, whether they are published or not. The documents may come from teaching and research institutions in France or abroad, or from public or private research centers.
L'archive ouverte pluridisciplinaire HAL, est destinée au dépôt et à la diffusion de documents scientifiques de niveau recherche, publiés ou non, émanant des établissements d'enseignement et de recherche français ou étrangers, des laboratoires publics ou privés. 


\title{
Pressure-dependent threshold in a granular flow: numerical modeling and experimental validation
}

\author{
L. Chupin ${ }^{\mathrm{a}}$, T. Dubois ${ }^{\mathrm{a}, *}$, M. Phan ${ }^{\mathrm{a}}$, O. Roche ${ }^{\mathrm{b}}$ \\ ${ }^{a}$ Université Clermont Auvergne, CNRS, LMBP, F-63000 Clermont-Ferrand, France \\ ${ }^{b}$ Université Clermont Auvergne, CNRS-IRD, OPGC, LMV, F-63000 Clermont-Ferrand, France
}

\begin{abstract}
Numerical simulations, performed with a new two-phase bi-projection scheme, of the collapse of columns of glass beads with aspect ratios equal to 0.7 and 2 over a horizontal plane are reported and comparison with experiments are presented. A level-set formulation for the Navier-Stokes equations is used, so that the interface between the granular material and the ambient air is tracked. The granular flow is modeled with a viscoplastic rheology, derived from the $\mu(I)$-rheology, resulting in a Drucker-Prager plasticity criterion combined with a spatio-temporal variable viscosity depending on the pressure and on the shear rate. The computational effort is reduced by using instead a constant viscosity. The dependence of the results upon the value of the constant viscosity, which has been reduced by two orders of magnitude, is very weak suggesting that these granular flows are mainly governed by the Drucker-Prager plasticity criterion. The rheology is formulated as a projection, allowing for an efficient computation of the plastic part of the stress tensor. Coulomb friction conditions are applied on the walls. The dynamics of the collapse and the morphology of the final deposit are accurately reproduced. Sensitivity of the results, with respect to the resolution and the basal friction coefficient, is also studied. During the collapse, the granular material consists of a basal deposit overlain by a flowing layer, which are separated by an interface that migrates upwards until the flowing layer is consumed. The time evolution of this static-mobile interface is quantified and a good agreement is found with experiments. To the best of our knowledge this is the first simulation of internal flow dynamics validated by experiments. We also report results obtained with a viscoplastic model where the dynamic pressure in the yield is replaced by the hydrostatic pressure, depending on the granular flow height. This model produces non-physically relevant solutions. Nevertheless, from a numerical point of view, it provides interesting and challenging test cases for two-phases viscoplastic simulations as the interface rolls up before the head of the granular mass falls on the bottom wall.
\end{abstract}

Keywords: Granular flow, Navier-Stokes equations, Multiphase flow, Rheology

\section{Introduction}

Dense flows of dry granular matter are common phenomena in the environment and industry. Modeling these flows is essential for testing rheological laws that govern the motion of the granular material, and it is also important in the context of assessment of natural hazards or control of industrial processes. Various configurations have been considered over the last decades to better understand the behavior of these flows (see the review 11). Among them, the collapse of dense and dry columns of granular materials over a horizontal plane, also known as the dambreak configuration, has been extensively studied both experimentally and numerically (see, for a recent review, 2]). This configuration is particularly well-suited for testing numerical simulations against laboratory experiments, from which detailed data on flow kinematics and deposit morphology can be acquired. The behavior of granular dambreak flows is well known. Once the granular column is released, the collapse, which lasts typically about one second in laboratory experiments, can be decomposed in four stages: a quick initial acceleration phase, followed by propagation of the front at constant velocity, further the flow decelerates and finally, while the front has reached the runout (maximum) distance, the granular flow moves very slowly until it stops. The granular flows during the collapse consist of

\footnotetext{
* Corresponding author

Email addresses: Laurent.Chupin@uca.fr (L. Chupin), Thierry.Dubois@uca.fr (T. Dubois), tran_duc_minh.phan@uca.fr (M. Phan), Olivier.Roche@uca.fr (O. Roche)
} 
a basal deposit, growing at a nearly constant rate, overlain by a moving part whose head slides on the bottom wall 3 . During the last stage, once the front has stopped, the basal deposit still grows slowly until it reaches the top and front of the mass. This internal dynamics, characterized by a static-mobile interface between the deposit and the moving layer, is quantified from experiments [3]. Also, the final deposit is characterized by its height, its runout distance, and its shape. Therefore, there are many available measured data on the collapse of granular flow, so that comparing numerical methods and/or different modeling approaches for this problem is appealing.

Numerical simulation of granular dambreak experiments are performed either by using depth-averaged models (see, for instance, [4, 5, 6, 7]), Discrete Element Methods (see, for instance, 8, 9]) or two-dimensional continuum models based on an elastoplastic constitutive relation with a Mohr-Coulomb yield rule [10] or on the $\mu(I)$-rheology proposed by Jop et al. [1] (see 12, 13, and 14]). Note that depth-averaged models based on the $\mu(I)$-rheology have been developped (see for instance 15). However, depth-averaged models are restricted to granular columns with aspect ratio less than unity and are not able to compute the dynamics of the static-mobile interface. Most of the above cited numerical studies focused on recovering scaling-laws that characterize the flow runout distance and that are provided by experiments (see, for instance [16]). In 13 and 14, detailed analyzes of the flow dynamics and comparisons of numerical simulations with experiments 17] have been first attempted. In [13, 14, the $\mu(I)$-rheology is reformulated as a continuum viscoplastic model. The yield criterion is a Drucker-Prager (DP) criterion with a threshold which is proportional to the pressure and a friction coefficient. The $\mu(I)$-rheology, written in the framework of viscoplastic models, has a spatio-temporal variable viscosity which depends on the norm of the strain-rate tensor and the pressure. This variability induces numerical difficulties, mainly in terms of computational efforts required, so that using instead a constant viscosity is appealing and, as mentioned in [12, provides a good approximation. Both models, with constant and variable viscosities, have been used in 13 and 14 . The same approach is followed in the present study. A constant viscosity model has the advantage that the only parameter that has to be adjusted is the viscosity while the $\mu(I)$-rheology depends on parameters that are not easily measurable. Note that developing a model with as less parameters as possible is advisable in a view to application to geophysical flows. Little differences were observed in [13, for viscosities in the range $[0.1,0.5] \mathrm{Pa}$ s, for collapse over a horizontal plane between results obtained with both models, and the results were in good agreement with experiments. In [14, the question of the numerical convergence of the results was partially addressed. Both models are known to be ill-posed in the sense that small-scale pressure oscillations may appear and lead to instabilities (see [18, 19, 14]). In 14, the authors decrease the mesh size down to $2 \times 10^{-3} \mathrm{~m}$ (approximately 3 times the diameter of the glass beads): short-wave instabilities take the form of bands of plug flows separated by thin zones of large gradients. Despite these small-scale oscillations, the numerical simulations remain stable and are able to predict and reproduce the main features of these granular flows.

In this paper, we propose to go further than earlier studies in the numerical simulation of the collapse of granular columns with the DP-model by studying the numerical convergence of the main flow characteristics (profile of the granular mass, kinematics of the collapse, ...), by estimating the influence of the viscosity and of the basal friction, and by analyzing the dynamics of the static-mobile interface. Two sets of experiments, which differ in the aspect ratio of the granular column and the size of the particles, are considered. The first one, with aspect ratio 0.7 , was already studied by 13 so that comparisons with their numerical results and with experiments can be performed. Numerical results for the second experiment, which was carried out by 20 and at an aspect ratio equal to 2 , are presented for the first time. In the present study, the rheological parameters, namely the internal and basal friction coefficients, are prescribed from the experiments. We have developed an efficient parallel code, so that we were able to decrease the mesh size until the numerical convergence of the profile of the granular mass as well as the collapse dynamics were obtained, which assessed the reliability of the results. The effect of the viscosity on the results could also be studied by decreasing its value by two orders of magnitude, down to $10^{-3} \mathrm{~Pa}$ s (hundred times smaller than in [13]) for the column with aspect ratio 0.7 .

As it was previously mentioned, while being able to reproduce the flow dynamics, incompressible models with DP yield stress are known to be mathematically ill-posed and are characterized by small-scale instabilities. Replacing the dynamic pressure by a hydrostatic pressure (HP-model) in the Drucker-Prager yield stress leads to a well-posed model (see [21]). The predictability of the HP-model has, up to now, never been investigated. We propose to evaluate the HP-model in the context of the collapse of granular columns. Simulations performed with the HP-model are also compared with the aforementioned experiments. Note that in [22, a well-posed compressible version of the $\mu(I)$-rheology has been proposed. Numerical methods for compressible or incompressible flows are fundamentally different. The main objective of this paper is to 
show that bi-projection schemes, developed for incompressible flows (see [23] and 24]), can be successfully applied to simulate the collapse of dry granular columns. Therefore, implementing the constitutive equations proposed in [22] is out of the scope of this paper.

In a viscoplastic rheology, the stress tensor is proportional to the strain-rate tensor when its strength is above the yield stress otherwise it is not prescribed. In order to handle this non-differentiable definition, a framework, which avoids the formalism of variational inequalities [25, was introduced in [23]: it relies on a projection formulation for the definition of the plastic part of the stress tensor. As for augmented Lagrangian 26] and Uzawa-like 27. methods, no regularization technique is needed in this case for the computation of rigid zones, which are regions where the strain-rate vanishes. By coupling this approach with classical fractional step methods to approximate solutions of the incompressible Navier-Stokes equations 28], an efficient bi-projection scheme for the time discretization of Bingham equations was proposed and studied in 23. Note that in this scheme, the plastic part of the stress tensor is computed with a fixed point procedure enjoying a geometrical convergence rate. The bi-projection scheme was further extended in [24] to viscoplastic fluids with spatially variable viscosity, density and yield stress. A version adapted from [24] is used in the present paper.

The surface of the granular mass during the collapse was treated as a free surface in [13] and [14, which means that the motion of the ambient medium was not computed. In this paper, we use the level-set method [29] to capture the interface between the granular mass and the ambient air. Interface tracking methods allow to handle a wider variety of problems and to use simple and efficient algorithms on Cartesian grids. In the level-set approach the interface is the set of zeros of a smooth function advected by the velocity field. However, for dambreak problems, the level-set method is known to encounter difficulties in accurately predicting the angle between the interface and a wall [30 and therefore the correct propagation of the front. We show in this paper that reducing the order of the schemes in the neighborhood of a boundary without adding ghost points and implementing friction boundary conditions permits us to produce very accurate numerical results in the context of the collapse of granular columns. Note that an alternative to the levelset method is the Volume of Fluid method, which has been successfully used by [31] to study viscoplastic dambreaks.

In this article, numerical simulations of the collapse of columns of glass beads, with aspect ratios 0.7 and 2, over horizontal plane are performed and compared with experimental results. Two continuum viscoplastic models with constant viscosity and pressure-dependent yield stress are considered. In section 2 , the mathematical models as well as the complementary boundary conditions and definitions of the yield stress threshold are given. The numerical methods are summarized in section 3 . The numerical results are analyzed and compared with laboratory experiments in section 4. Two experiments are considered and they differ in the aspect ratio of the granular column and the size of the glass beads. The flow dynamics and the profile of the granular mass obtained with the DP-model are compared with experiment. Sensibilities of the numerical results upon the resolution, values of the constant viscosity and/or of the basal friction coefficient are studied. For the second experiment, with an initial column with aspect ratio 2, numerical results are presented for the first time. In this case, the static-mobile interface, which characterizes the internal dynamics of the granular flow, is analyzed. For both experiments, simulations performed with the HP-model are also presented.

\section{Mathematical modeling}

\subsection{Conservation laws and rheology}

We consider the collapse of a granular mass over a horizontal rigid surface. The mathematical model is written in two-dimension and involves two components: a viscoplastic part corresponding to the granular mass, and a Newtonian part modeling the surrounding air. The flow is assumed to be incompressible with variable density (describing the two components) so that the mass and momentum conservation laws read

$$
\begin{aligned}
& \rho\left(\partial_{t} \boldsymbol{u}+\boldsymbol{u} \cdot \boldsymbol{\nabla} \boldsymbol{u}\right)=\operatorname{div} \boldsymbol{T}+\rho \boldsymbol{g}, \\
& \operatorname{div} \boldsymbol{u}=0, \\
& \partial_{t} \rho+\boldsymbol{u} \cdot \boldsymbol{\nabla} \rho=0,
\end{aligned}
$$

where $\boldsymbol{u}$ is the material velocity, $\boldsymbol{g}$ an external force (gravity), $\rho$ the mass density, and $\boldsymbol{T}$ the total stress

tensor. The pressure is given by $p=-\frac{1}{3} \operatorname{tr} \boldsymbol{T}$, so that the deviatoric stress $\boldsymbol{T}^{\prime}\left(\boldsymbol{T}=-p \boldsymbol{I} \boldsymbol{d}+\boldsymbol{T}^{\prime}\right)$ has to be prescribed in order to close equations (1) $-(3)$. 
Granular material, like viscoplastic fluids, flow only if the stress exceeds a threshold, the yield stress; otherwise they do not deform and behave like solids. Classical viscoplastic fluids of Bingham type, have a constant yield stress. In order to account for internal friction, the yield stress of granular materials is proportional to the pressure (see 32 for instance). This peculiar behavior is modeled by the Drucker-Prager plasticity criterion

$$
\left|\boldsymbol{T}^{\prime}\right| \leq \mu p
$$

in regions where no deformation occur, namely where $|\boldsymbol{D} \boldsymbol{u}|=0$. Here, the norm is the modified Froebenius norm $|\boldsymbol{\lambda}|^{2}=\frac{1}{2} \sum_{i, j} \boldsymbol{\lambda}_{i j}^{2}$ for any tensor $\boldsymbol{\lambda}, \boldsymbol{D}(\boldsymbol{u})=\frac{1}{2}\left(\boldsymbol{\nabla} \boldsymbol{u}+(\boldsymbol{\nabla u})^{t}\right)$ is the strain-rate tensor, and $\mu$ is a friction coefficient. In regions where the stress exceeds the threshold, the deviatoric stress tensor is proportional to the strain-rate tensor,

$$
\boldsymbol{T}^{\prime}=\mu p \frac{\boldsymbol{D u}}{|\boldsymbol{D u}|},
$$

so that $\frac{\mu p}{|\boldsymbol{D} \boldsymbol{u}|}$ is an effective viscosity of the granular material depending on the pressure and the shear rate. The $\mu(I)$-rheology is based on the constitutive law (5). In [11, Jop et al introduce a dependence of the friction coefficient of the granular material in terms of the (non-dimensional) inertial number, which is the square root of the Savage number 33] or of the Coulomb number 34] and can be interpreted as the ratio of an inertial microscopic timescale of particle rearrangement $d / \sqrt{p / \rho_{s}}$, where $d$ and $\rho_{s}$ are respectively the particle diameter and density, and a macroscopic deformation timescale $\left(|\boldsymbol{D} \boldsymbol{u}|^{-1}\right)$. The inertial number $I$ is defined by

$$
I=2|\boldsymbol{D}(\boldsymbol{u})| d \sqrt{\frac{\rho_{\mathrm{s}}}{p}}
$$

and the friction law, compatible with experimental measurements, proposed in [11] writes

$$
\mu(I)=\mu_{s}+\frac{\mu_{\infty}-\mu_{s}}{1+I_{0} / I},
$$

where $I_{0}$ is a dimensionless number, $\mu_{s}=\tan (\alpha), \alpha$ being the internal friction angle depending on the granular material, and $\mu_{\infty} \geq \tan (\alpha)$ is an asymptotic value of the friction coefficient for large inertial numbers. In this formalism, the deviatoric stress is defined by

$$
\begin{cases}\boldsymbol{T}^{\prime}=\mu(I) p \frac{\boldsymbol{D}(\boldsymbol{u})}{|\boldsymbol{D}(\boldsymbol{u})|} & \text { if } \boldsymbol{D}(\boldsymbol{u}) \neq 0, \\ \left|\boldsymbol{T}^{\prime}\right| \leq \tan (\alpha) p & \text { if } \boldsymbol{D}(\boldsymbol{u})=0 .\end{cases}
$$

As pointed out in [11, the yield criterion (9) of the $\mu(I)$-rheology is of Drucker-Prager type. By reporting the definitions (6) and (7), for $I$ and $\mu(I)$ respectively, in (8), we can rewrite the expression of the tensor $\boldsymbol{T}^{\prime}$ in regions where $\boldsymbol{D}(\boldsymbol{u}) \neq 0$ as

$$
\boldsymbol{T}^{\prime}=\frac{\left(\mu_{\infty}-\mu_{s}\right) p}{|\boldsymbol{D}(\boldsymbol{u})|+\frac{I_{0} \sqrt{p}}{2 d \sqrt{\rho_{s}}}} \boldsymbol{D}(\boldsymbol{u})+\tan (\alpha) p \frac{\boldsymbol{D}(\boldsymbol{u})}{|\boldsymbol{D}(\boldsymbol{u})|}
$$

or equivalently

$$
\boldsymbol{T}^{\prime}=2 \eta(|\boldsymbol{D}(\boldsymbol{u})|, p) \boldsymbol{D}(\boldsymbol{u})+\tan (\alpha) p \frac{\boldsymbol{D}(\boldsymbol{u})}{|\boldsymbol{D}(\boldsymbol{u})|}
$$

with

$$
\eta(|\boldsymbol{D}(\boldsymbol{u})|, p)=\frac{\left(\mu_{\infty}-\tan (\alpha)\right) p}{2|\boldsymbol{D}(\boldsymbol{u})|+I_{0} \sqrt{p} / d \sqrt{\rho_{\mathrm{s}}}} .
$$

Rewriting the $\mu(I)$-rheology as a viscoplastic model allows to treat the non-differentiable definition of the Drucker-Prager yield criterion with specific algorithms. In [13, the numerical method relies on the Lagrangian augmented method while we use a bi-projection scheme. The main objective of the present work is to apply and show the ability of bi-projection schemes to accurately predict the collapse of dry granular columns.

In the form 10], the $\mu(I)-$ rheology can be interpreted as a viscoplastic model with a Drucker-Prager yield criterion and a spatio-temporal variable viscosity. This variability may induce very small local values of the viscosity resulting in a high computational cost. It is therefore interesting, from a computational 
point of view, to consider a version of the rheology 110$)$ with a constant viscosity instead of $\eta(\mid \boldsymbol{D}(\boldsymbol{u}), p)$ given by (11). Both cases will be considered in the present study to model the granular material. Note that in [13, very little differences were found between results obtained with a constant viscosity and the spatio-temporal variable viscosity prescribed by the $\mu(I)-$ rheology. In the sequel, the $\mu(I)-$ rheology will be mainly used to predict the order of magnitude of $\eta(\mid \boldsymbol{D}(\boldsymbol{u}), p)$. Moreover, we will show that the main features of the granular mass during the collapse are recovered with much smaller values of the viscosity indicating that the dynamics of these flows are mainly governed by the Drucker-Prager plasticity criterion (4).

To take into account the presence of the two phases, we will assume that initially the density $\rho$ takes exactly two constant values: that of the air $\rho_{\mathrm{f}}$ and that of the granular medium $\rho_{\mathrm{s}}$. We will assume that this property will be satisfied throughout the experiment (from a numerical point of view, this property will be ensured using a level-set method). Obviously, both the viscosity and the yield stress also depend on $\rho$, so that the deviatoric stresses, for both phases, write:

$\checkmark$ in the Newtonian phase $\left(\rho=\rho_{\mathrm{f}}\right)$

$$
\boldsymbol{T}^{\prime}=2 \eta_{f} \boldsymbol{D}(\boldsymbol{u})
$$

with $\eta_{\mathrm{f}}$ the air dynamic viscosity.

$\checkmark$ and in the granular material $\left(\rho=\rho_{\mathrm{s}}\right)$

$$
\begin{cases}\boldsymbol{T}^{\prime}=2 \eta_{\mathrm{s}} \boldsymbol{D}(\boldsymbol{u})+\tan (\alpha) p \frac{\boldsymbol{D}(\boldsymbol{u})}{|\boldsymbol{D}(\boldsymbol{u})|} & \text { if } \boldsymbol{D}(\boldsymbol{u}) \neq 0, \\ \left|\boldsymbol{T}^{\prime}\right| \leq \tan (\alpha) p & \text { if } \boldsymbol{D}(\boldsymbol{u})=0 .\end{cases}
$$

where $\eta_{\mathrm{s}}=\eta_{0}$, with $\eta_{0}$ is a prescribed (constant) value, or $\eta_{s}=\eta(|\boldsymbol{D}(\boldsymbol{u})|, p)$ given by (11).

Note that in the granular medium a Drucker-Prager plasticity criterion is used with the yield depending on the pressure and the static internal friction angle while the Newtonian fluid has no plastic behavior.

In some cases, it may be interesting to replace, in the expression of yield stress, the pressure $p$ by a hydrostatic pressure denoted $p_{\text {hyd }}$ and depending on the weight above a point. This choice can be intuitive when considering thin flows or more generally when the flows are mainly driven by the pressure and the gravity forces: in that cases, the momentum equation (1) reduces to $\nabla p_{\text {hyd }}=\rho \boldsymbol{g}$. Consequently, we also propose the following model instead of $120-13$ :

$$
\begin{cases}\boldsymbol{T}^{\prime}=2 \eta_{0} \boldsymbol{D}(\boldsymbol{u})+\tan (\alpha) p_{\text {hyd }} \frac{\boldsymbol{D}(\boldsymbol{u})}{|\boldsymbol{D}(\boldsymbol{u})|} & \text { if } \boldsymbol{D}(\boldsymbol{u}) \neq 0, \\ \left|\boldsymbol{T}^{\prime}\right| \leq \tan (\alpha) p_{\text {hyd }} & \text { if } \boldsymbol{D}(\boldsymbol{u})=0,\end{cases}
$$

where $p_{\text {hyd }}(x, z)=\int_{z}^{\max \left\{y ; \rho(x, y)=\rho_{s}\right\}} \rho g$, where $g$ is the gravitational constant. One of the objectives of this work is to apply this rheology. Note that only the case of a constant viscosity $\eta_{0}$ will be considered. In the remaining of the paper, the model based on $[12)-(13)$ will be named the DP-model while HP-model will be used to designate the one based on (14) 15 .

Let us now introduce a generic form, which will be useful in the sequel, for the definition of the above constitutive laws $12-13$ and $14-15$. Namely, we write

$$
\begin{cases}\boldsymbol{T}^{\prime}=2 \eta \boldsymbol{D}(\boldsymbol{u})+\kappa \frac{\boldsymbol{D}(\boldsymbol{u})}{|\boldsymbol{D}(\boldsymbol{u})|} & \text { if } \boldsymbol{D}(\boldsymbol{u}) \neq 0 \\ \left|\boldsymbol{T}^{\prime}\right| \leq \kappa & \text { if } \boldsymbol{D}(\boldsymbol{u})=0\end{cases}
$$

where

$$
\begin{aligned}
& \text { if } \rho=\rho_{\mathrm{f}} \quad \eta=\eta_{\mathrm{f}} \quad \text { and } \quad \kappa=0 \text {, } \\
& \text { if } \rho=\rho_{\mathrm{s}} \quad\left\{\begin{array}{llll}
\eta=\eta_{\mathrm{s}} & \text { and } & \kappa=\tan (\alpha) p & \text { (DP-model) } \\
\eta=\eta_{\mathrm{s}} & \text { and } & \kappa=\tan (\alpha) p_{\text {hyd }} & \text { (HP-model) }
\end{array}\right.
\end{aligned}
$$

Let us recall that $\eta_{\mathrm{s}}$ for the DP-model in 19 has either a constant prescribed value $\eta_{0}$ or is the spatiotemporal variable viscosity predicted by the $\mu(I)$-rheology and given by (11). 


\subsection{Boundary conditions}

The equations (1)-(3) are supplemented with boundary conditions. The experiments considered in this paper have been conducted in a narrow and elongated channel formed by plexiglass walls. As we restrict our study to a bi-dimensional model, the computational domain $\Omega$ is therefore a rectangular channel. The bottom and back walls, namely the solid (plexiglass) boundaries, are denoted by $\partial \Omega_{\mathrm{w}}$ so that $\partial \Omega_{\mathrm{a}}=\partial \Omega \backslash \partial \Omega_{\mathrm{w}}$ is the remaining part of the domain boundary. The granular medium slides on $\partial \Omega_{\mathrm{w}}$ while the surrounding air is free to slide or exit on $\partial \Omega_{\mathrm{a}}$. Different boundary conditions should be imposed on both parts of $\partial \Omega$.

In any case, we will note by $\boldsymbol{n}$ the outgoing unit normal to the domain. In practice, we decompose the velocity $\boldsymbol{u}$ at the boundary into normal velocity $u_{n}=\boldsymbol{u} \cdot \boldsymbol{n}$ and tangent velocity $\boldsymbol{u}_{t}=\boldsymbol{u}-u_{n} \boldsymbol{n}$. In the same way, we introduce the following notations for the stress: $T_{n}=(\boldsymbol{T} \cdot \boldsymbol{n}) \cdot \boldsymbol{n}$ and $\boldsymbol{T}_{t}=\boldsymbol{T} \cdot \boldsymbol{n}-T_{n} \boldsymbol{n}$.

For consistency with the divergence-free condition, the normal velocity $u_{n}$ is enforced to be zero over the whole domain boundary, that is

$$
u_{n}=0 \quad \text { on } \partial \Omega .
$$

On the bound $\partial \Omega_{\mathrm{a}}$, free-slip boundary conditions are applied, so that 20 is supplemented with

$$
\partial_{n} \boldsymbol{u}_{t}=0 .
$$

On the back and bottom walls $\partial \Omega_{\mathrm{w}}$, we take into account friction with the granular medium so that, if $\rho=\rho_{s}$ the boundary conditions must be written

$$
\begin{cases}\boldsymbol{T}_{t}=-\kappa_{\mathrm{b}} \frac{\boldsymbol{u}_{t}}{\left|\boldsymbol{u}_{t}\right|} & \text { if } \boldsymbol{u}_{t} \neq \mathbf{0} \\ \left|\boldsymbol{T}_{t}\right| \leq \kappa_{\mathrm{b}} & \text { if } \boldsymbol{u}_{t}=\mathbf{0}\end{cases}
$$

where $\kappa_{\mathrm{b}}=\tan \left(\alpha_{b}\right)\left(-T_{n}\right)^{+}$. The "+" superscript stands for the positive part, namely $a^{+}=\max (0, a)$. The coefficient $\tan \left(\alpha_{b}\right)$ is the Coulomb friction coefficient and $\alpha_{b}$ is the basal friction angle.

Sliding of air is allowed on $\partial \Omega_{\mathrm{w}}$ by applying Navier boundary conditions, that is, if $\rho=\rho_{f}$,

$$
\eta \frac{\partial \boldsymbol{u}_{t}}{\partial n}+\eta_{\mathrm{b}} \boldsymbol{u}_{t}=0 .
$$

The main advantage of Navier conditions is that, depending upon the value of the parameter $\eta_{\mathrm{b}}$, they allow to impose Dirichlet $\left(\eta_{\mathrm{b}}=\infty\right)$, mixed $\left(0<\eta_{\mathrm{b}}<\infty\right)$ or Neumann $\left(\eta_{\mathrm{b}}=0\right)$ boundary conditions. In most numerical simulations presented in the paper, the value $\eta_{\mathrm{b}}=1$ will be used.

\section{Numerical method}

\subsection{Level-set approach}

The interface between the air and the granular medium is tracked with a level-set function $\phi$, so that the interface $\Gamma$ is the set of points where $\phi$ vanishes. The level-set function is a smooth function of the position

$\boldsymbol{x}$ : the signed distance function from the interface is commonly used; see [35] and the reference therein. The physical density is smeared out at the interface, so that we define

$$
\rho(\phi)=\rho_{\mathrm{f}} H_{\epsilon}(\phi)+\rho_{\mathrm{s}}\left(1-H_{\epsilon}(\phi)\right),
$$

where $H_{\epsilon}(\phi)$ is the regularized Heaviside function

$$
H_{\epsilon}(\phi)= \begin{cases}0 & \text { if } \phi<-\epsilon \\ \frac{1}{2}\left(1+\frac{\phi}{\epsilon}+\sin \left(\frac{\pi \phi}{\epsilon}\right) / \pi\right) & \text { if }|\phi| \leq \epsilon \\ 1 & \text { if } \phi>\epsilon\end{cases}
$$

In order to ensure continuity of the tangential shear stress, the viscosity $\eta(\phi)$ is a harmonic mean of $\eta_{\mathrm{s}}$ and $\eta_{\mathrm{f}}$

$$
\frac{1}{\eta(\phi)}=\frac{\theta(\phi)}{\eta_{\mathrm{s}}}+\frac{(1-\theta(\phi))}{\eta_{\mathrm{f}}}
$$


where the height fraction $\theta(\phi)$ is locally defined (as in [36]), for any $\boldsymbol{x} \in \Omega$, by

$$
\theta(\phi(\boldsymbol{x}))=\frac{\int_{K(\boldsymbol{x})} \phi^{+}(\boldsymbol{y}) d \boldsymbol{y}}{\int_{K(\boldsymbol{x})}|\phi(\boldsymbol{y})| d \boldsymbol{y}}
$$

where $K(\boldsymbol{x})$ is a neighborhood of $\boldsymbol{x}$, so that $|K(\boldsymbol{x})|$ is of the order of the mesh size. Note that $\theta(\phi(\boldsymbol{x}))=1$ if $K(\boldsymbol{x}) \subset\{\boldsymbol{y} \in \Omega ; \phi(\boldsymbol{y})>0\}, \theta(\phi(\boldsymbol{x}))=0$ if $K(\boldsymbol{x}) \subset\{\boldsymbol{y} \in \Omega ; \phi(\boldsymbol{y})<0\}$ and $\theta(\boldsymbol{x}) \in(0,1)$ otherwise.

The yield stress $\kappa(\phi)$ is treated as a sharp discontinuity according to the sign of the levelset function, namely

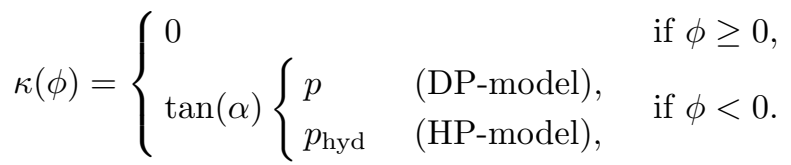

In the level-set formulation (see [29, 37, 38]), $\phi$ is advected by the velocity so that mass conservation equation (3) is replaced by a transport equation. In this context, the level-set formulation modeling a column of granular medium sliding into ambient air writes

$$
\begin{aligned}
& \partial_{t} \phi+\operatorname{div}(\boldsymbol{u} \phi)=0, \\
& \rho(\phi)\left(\partial_{t} \boldsymbol{u}+\operatorname{div}(\boldsymbol{u} \otimes \boldsymbol{u})\right)+\nabla p=-\rho(\phi) g \boldsymbol{e}_{2}+\operatorname{div}(2 \eta(\phi) \boldsymbol{D}(\boldsymbol{u})+\kappa(\phi) \boldsymbol{S}), \\
& \operatorname{div} \boldsymbol{u}=0,
\end{aligned}
$$

where $g$ is the gravitational constant, $\boldsymbol{e}_{2}$ is the vertical unit vector and $\boldsymbol{S}$ is the plastic part of the stress tensor that will be rigorously defined in section 3.2. Equations (26)- $(28)$ are supplemented by the boundary conditions (20) and (21) on $\partial \Omega_{a}$. On the back and bottom walls $\partial \Omega_{\mathrm{w}}$, boundary conditions (20) and (24) are applied where $\phi \geq 0$ while 20), 22) and (23) are enforced where $\phi<0$. Note that nonlinear terms are written in conservative form in level-set and momentum equations. Also, surface tension forces are not taken into account.

The transport equation (26) is discretized with a finite volume WENO scheme of order 5 [39, 40] in space combined with TVD RK3 scheme for the time discretization [41]. Even if the discrete level-set function is initialized as the signed distance from the interface, it will not remain a distance function and, as it is suggested in [29], a redistancing algorithm needs to be applied periodically in time. A classical approach consists in solving numerically a Hamilton-Jacobi equation. Stationary solutions of this equation are distance functions and they share their interface with the level-set function. In this paper, the redistancing algorithm given by [42] is used. It relies on a TVD RK2 method as time marching scheme and on a ENO2 finitedifference scheme for the spatial resolution. Also, we have implemented the subcell fix resolution detailed in [42, which modifies the ENO2 scheme near the interface in order to avoid artificial displacement of the interface during the redistancing iterative process.

\subsection{Bingham projection}

According to the rheological law (16)-(17), the plastic part $\boldsymbol{S}$ of the total stress tensor is simply given by $\boldsymbol{S}=\boldsymbol{D}(\boldsymbol{u}) /|\boldsymbol{D}(\boldsymbol{u})|$, which is not well defined in the rigid zone, i.e. where the strain-rate tensor vanishes. In this case, the constraint $|\boldsymbol{S}| \leq 1$ should be added. A convenient way to define $\boldsymbol{S}$ is to use the formalism introduced in 23] (see also 24]), that is

$$
\boldsymbol{S}=P(\boldsymbol{S}+r \kappa(\phi) \boldsymbol{D}(\boldsymbol{u})), \quad \text { for any } r>0,
$$

where $P$ is a projection operator which is explicitly defined by

$$
P(\boldsymbol{L})= \begin{cases}\frac{\boldsymbol{L}}{|\boldsymbol{L}|} & \text { if }|\boldsymbol{L}|>1, \\ \boldsymbol{L} & \text { if }|\boldsymbol{L}| \leq 1,\end{cases}
$$

for any traceless symmetric tensor $\boldsymbol{L}$. Note that the boundary condition $222-23)$ can be written similarly

$$
\boldsymbol{T}_{t}=-\kappa_{\mathrm{b}} \boldsymbol{s}_{t} \quad \text { with } \boldsymbol{s}_{t}=P_{b}\left(\boldsymbol{s}_{t}+r \kappa_{\mathrm{b}} \boldsymbol{u}_{t}\right), \text { for any } r>0,
$$

where $P_{b}(\boldsymbol{v})=\left\{\begin{array}{ll}\boldsymbol{v} /|\boldsymbol{v}| & \text { if }|\boldsymbol{v}|>1, \\ \boldsymbol{v} & \text { if }|\boldsymbol{v}| \leq 1,\end{array}\right.$ for any vector $\boldsymbol{v}$. The complete system of equations is then formed by 26 - 28 supplemented with 29 - 230 .

Note that this approach based on projection operators avoids the use of a regularization technique to compute the rigid (plastic) zones, i.e. areas where the strain-rate tensor vanishes. 


\subsection{Chorin-Temam algorithm}

Let $\delta t>0$ be the time step. Assuming that the approximations $\left(\boldsymbol{u}^{n}, \phi^{n}, p^{n}, \boldsymbol{S}^{n}, \boldsymbol{s}_{t}^{n}\right)$ of $\left(\boldsymbol{u}, \phi, p, \boldsymbol{S}, \boldsymbol{s}_{t}\right)$ at discrete time $t_{n}=n \delta t$ are known, $\phi^{n+1}$ is computed as described in section 3.1. Note that $\boldsymbol{u}^{n}$, which is a solenoidal velocity field, is used to advect $\phi^{n}$ and then compute $\phi^{n+1}$. We set: $\rho^{n+1}=\rho\left(\phi^{n+1}\right), \eta^{n+1}=$ $\eta\left(\phi^{n+1}\right), \kappa^{n+1}=\kappa\left(\phi^{n+1}\right)$. The computational domain is divided into $\Omega^{n+1,+}=\left\{\boldsymbol{x} \in \Omega ; \phi^{n+1}(\boldsymbol{x})>0\right\}$ and $\Omega^{n+1,-}=\left\{\boldsymbol{x} \in \Omega ; \phi^{n+1}(\boldsymbol{x})<0\right\}$. The momentum equation is discretized with a second-order fractional time stepping scheme inspired from the pioneering Chorin-Temam algorithm [43, 44. This approach allows to decouple velocity and pressure in a step predicting a non-solenoidal velocity field followed by an update of the pressure used to project the estimated velocity onto the space of divergence-free velocity field. Following [23] and 24], the prediction step reads

$$
\begin{aligned}
& \rho^{n+1}\left(\frac{3 \tilde{\boldsymbol{u}}^{n+1}-4 \boldsymbol{u}^{n}+\boldsymbol{u}^{n-1}}{2 \delta t}+2 \operatorname{div}\left(\boldsymbol{u}^{n} \otimes \boldsymbol{u}^{n}\right)-\operatorname{div}\left(\boldsymbol{u}^{n-1} \otimes \boldsymbol{u}^{n-1}\right)\right) \\
& \quad+\boldsymbol{\nabla} p^{n}=-\rho^{n+1} g \boldsymbol{e}_{2}+\operatorname{div}\left(2 \eta^{n+1} \boldsymbol{D}\left(\tilde{\boldsymbol{u}}^{n+1}\right)+\kappa^{n+1} \boldsymbol{S}^{n+1}\right) \\
& \boldsymbol{S}^{n+1}=P\left(\boldsymbol{S}^{n+1}+r \kappa^{n+1} \boldsymbol{D}\left(\tilde{\boldsymbol{u}}^{n+1}\right)+\theta\left(\boldsymbol{S}^{n}-\boldsymbol{S}^{n+1}\right)\right), \quad \text { for any } r>0, \theta \in(0,1), \\
& u_{n}^{n+1}=0, \text { on } \partial \Omega, \quad \partial_{n} \boldsymbol{u}_{t}^{n+1}=0, \text { on } \partial \Omega_{\mathrm{a}}, \\
& \boldsymbol{T}_{t}^{n+1}=-\kappa_{\mathrm{b}}^{n+1} \boldsymbol{s}_{t}^{n+1}, \boldsymbol{s}_{t}^{n+1}=P_{b}\left(\boldsymbol{s}_{t}^{n+1}+r_{\mathrm{b}} \kappa_{\mathrm{b}}^{n+1} \tilde{\boldsymbol{u}}_{t}^{n+1}+\theta\left(\boldsymbol{s}_{t}^{n}-\boldsymbol{s}_{t}^{n+1}\right)\right), \text { for any } r_{\mathrm{b}}>0, \text { on } \partial \Omega_{\mathrm{w}}^{n+1,-}, \\
& \eta \partial_{n} \boldsymbol{u}_{t}^{n+1}+\eta_{b} \boldsymbol{u}_{t}^{n+1}=0, \text { on } \partial \Omega_{\mathrm{w}}^{n+1,+},
\end{aligned}
$$

where $\kappa_{\mathrm{b}}^{n+1}=\tan \left(\alpha_{\mathrm{b}}\right)\left(-T_{n}^{n+1}\right)^{+}$. Then, the projection step reads

$$
\begin{aligned}
& \rho^{n+1}\left(\frac{3\left(\boldsymbol{u}^{n+1}-\tilde{\boldsymbol{u}}^{n+1}\right)}{2 \delta t}\right)+\boldsymbol{\nabla}\left(p^{n+1}-p^{n}\right)=0, \\
& \operatorname{div} \boldsymbol{u}^{n+1}=0, \quad\left(\boldsymbol{u}^{n+1}-\tilde{\boldsymbol{u}}^{n+1}\right) \cdot \boldsymbol{n}=0 \text { on } \partial \Omega
\end{aligned}
$$

In (31), $\left(\tilde{\boldsymbol{u}}^{n+1}, \boldsymbol{S}^{n+1}, \boldsymbol{s}_{t}^{n+1}\right)$ are nonlinearly coupled. Due to the non-differentiability of the viscoplastic rheology, a Newton algorithm can not be directly applied. Therefore, we use a Picard fixed point procedure to solve (31). The pseudo-relaxation terms depending on the parameter $\theta$ added in the projectors $P$ and $P_{b}$ allow for a geometric convergence with common ratio $(1-\theta)$ of the fixed point iterations; see [23] and [24] for details. The projection step (32) consists essentially in solving a variable coefficients elliptic equation for $p^{n+1}$ followed by a correction applied to the non solenoidal velocity $\tilde{\boldsymbol{u}}^{n+1}$ in order to obtain $\boldsymbol{u}^{n+1}$, which is divergence-free (up to the computer accuracy).

\subsection{Numerical implementation}

The aim of this paper being to numerically simulate, with a bi-dimensional model, the collapse of columns of granular matter in a rectangular channel, $\Omega=(0, \mathcal{L}) \times(0, \mathcal{H})$ is used as computational domain. The initial granular column is defined by its length $L_{c}$ and height $H_{c}$ as it can be seen on figure 1 representing the configuration before release.

The computational domain is discretized in $\frac{\mathcal{L} n}{\mathcal{H}} \times n$ Cartesian mesh cells. Let us denote by $h=\frac{\mathcal{H}}{n}$ the mesh size. As in the classical MAC scheme for the incompressible Navier-Stokes equations [45] the discrete velocity unknowns $\boldsymbol{u}_{i j}$ are located at the midpoint of cell edges. The discrete pressure $p_{i j}$ and level-set function $\phi_{i j}$ are placed at the center of the mesh cell $K_{i j}$. All components of the plastic tensor $\boldsymbol{S}$ are also discretized at the center of the mesh cell. This choice is arbitrary but allows to update all tensor components, through the local projection (29), at the same mesh locations [23].

The implementation has been done in a F90/MPI code previously written for one phase Bingham flows and used for simulating flows in a lid-driven cavity at Reynolds number up to 200000 and Bingham number equals to 100 [23. The PETSc library [46, 47] is used to solve linear systems and to manage data on structured grids. The communications between the processes are explicitly written with MPI subroutines.

\section{Results and comparisons with laboratory experiments}

\subsection{Collapse of a dry granular column with aspect ratio 0.7 over a rough surface}

\subsubsection{Experimental setup}

We first consider one experiment in [17] consisting of the collapse of a dry granular column (glass beads with diameter $d \approx 0.7 \pm 0.1 \mathrm{~mm}$ ) in a $10 \mathrm{~cm}$ wide channel between plexiglass walls. The horizontal plane 


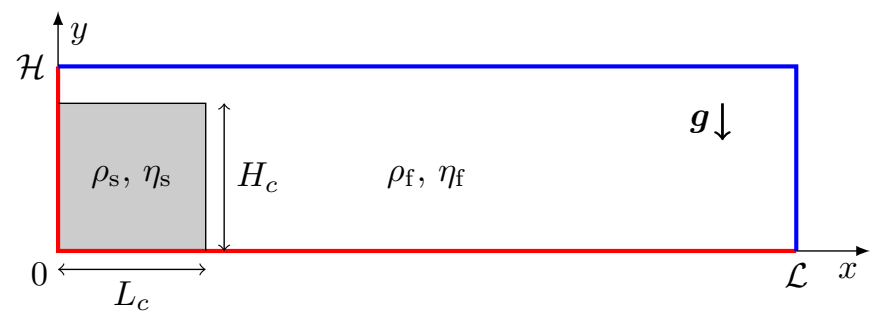

Figure 1: Configuration for the collapse of a granular column initially at rest. The blue part of the domain boundary corresponds to $\partial \Omega_{\mathrm{a}}$ (free air) while the red one corresponds to $\partial \Omega_{\mathrm{w}}$ (solid walls).

is $3 \mathrm{~m}$ long and is roughened by a layer of glass beads (with same diameter) glued on its surface. The granular medium in a reservoir has a length $L_{c}=20 \mathrm{~cm}$ and a height $H_{c}=14 \mathrm{~cm}$, resulting in an aspect ratio $H_{c} / L_{c}=0.7$, and is retained by a gate, which is opened at time $t=0 \mathrm{~s}$. The particle density is $\rho_{\mathrm{p}}=$ $2500 \mathrm{~kg} \mathrm{~m}^{-3}$ so that with a volume fraction of 0.62 the apparent flow density is $\rho_{\mathrm{s}}=0.62 \rho_{\mathrm{p}}=1550 \mathrm{~kg} \mathrm{~m}^{-3}$.

\subsubsection{Validation of the implementation of the DP-rheology}

In order to validate the numerical implementation of the DP-model previously described, we first confront our results with the experiment and with those published in [13. Indeed in [13, numerical simulations corresponding to this experiment have been performed with a continuum model using the $\mu(I)-$ rheology, reformulated, as in equations (10)- 11], in the framework of a viscoplastic model.

Let us highlight the major differences between our numerical implementation of the DP-rheology and the one used in [13. The presence of the ambient gas (air) is not accounted for in [13] and equations of motions are only solved in the viscoplastic fluid domain, i.e containing the granular material. In our study, both the granular and the ambient flow are modeled through a level-set formulation (see section 3). Another difference is the presence of the gate, which is not modeled in our numerical simulations, but is accounted for with a time shift of $-0.03 \mathrm{~s}$, which is half the lifting time of the gate. In order to compare our numerical results with those in [13, the same rheological parameters are used and the reader is referred to [13] for explanations of the chosen values. The friction coefficient of the glass beads on the plexiglass wall at the back of the channel is $\kappa_{\mathrm{b}}=\tan \left(10.5^{\circ}\right)=0.18$ while $\kappa_{\mathrm{b}}=\tan \left(25.5^{\circ}\right)=0.48$ is used on the rough horizontal plane. The static friction coefficient in the yield $(19)$ is $\tan \left(25.5^{\circ}\right)=0.48$.

In order to estimate the viscosity $\eta_{\mathrm{s}}$, we have first performed a simulation on a coarse mesh $(h=0.2 / 64=$ $31.25 \mathrm{~mm}$ ) with the $\mu(I)$-rheology, namely with $\eta_{s}=\eta(\boldsymbol{D} \boldsymbol{u}, p)$ computed with 11$)$ where $\mu_{\infty}=0.73$ and $I_{0}=0.279$ (as in [13]). The viscosity $\eta(\boldsymbol{D u}, p)$ is bounded from above by $1.2 \mathrm{~Pa}$ s and decreases with height (see figure 2). During the collapse, in most of the granular mass, the $\mu(I)$-viscosity takes values above 0.5 $\mathrm{Pa}$ s. This region is overlain by a $3 \mathrm{~cm}$ deep layer where $\eta(\boldsymbol{D u}, p)<0.5 \mathrm{~Pa} \mathrm{~s}$. As it was already mentioned, the spatio-temporal variations of the viscosity in the $\mu(I)$-rheology induces large computational times, so that performing numerical simulations on finer meshes with a constant viscosity $\eta_{\mathrm{s}}=\eta_{0}$ is preferable. By assuming that the strength of the plastic part in the DP-model dominates over the viscous part in the bottom part of the granular mass, we retained the value $\eta_{0}=0.5 \mathrm{~Pa}$ s for the simulations presented hereafter. The question of the appropriate value of the viscosity will be further investigated by performing simulations with smaller values of $\eta_{0}$ (see section 4.1.4).

In figure 3, the granular mass at different times during its collapse is plotted and compared with the profile of the experiment and of the numerical results presented in [13. Note that here, the numerical simulation is performed by using 256 mesh points in the vertical direction, which corresponds to a mesh size $h=0.2 / 256=0.78125 \mathrm{~mm}$ similar to the size of the glass beads used in the experiment; in [13], the resolution was coarser with the mesh size in the interval $[3.3,10] \mathrm{mm}$. We observe on figure 3 that the height of the granular column on the back wall of the reservoir is maintained at its initial value during the whole collapse as in the experiment while it is underestimated by nearly $10 \%$ in 13 . The plateau, corresponding to the left upper part of the granular mass (and touching the back plexiglass wall), found in the experiment is very well captured by our numerical simulation. In both simulations, the fronts of the granular masses, corresponding to the most advanced part of the sliding material located on the horizontal plate, are slightly ahead of the experimental front during the first half period of the collapse, that is for $t \leq 0.42 \mathrm{~s}$. This may be explained by the presence of the gate, which is not properly accounted for in the numerical methods so that small differences on the dynamics are observed at the beginning of the collapse process, namely the acceleration phase. 

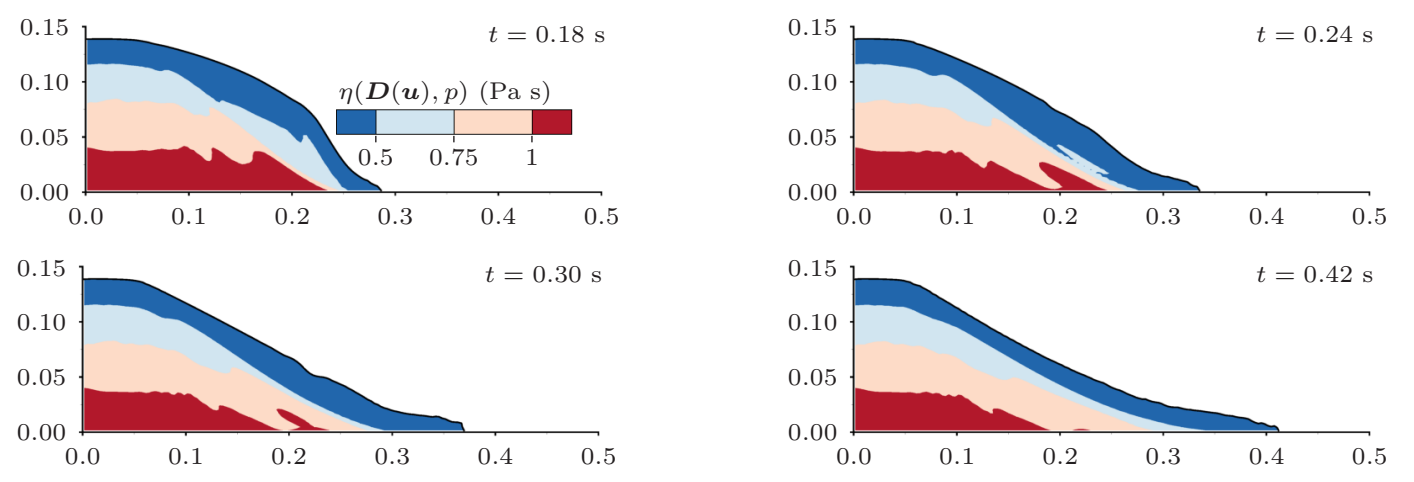

Figure 2: Values of the viscosity $\eta(\boldsymbol{D}(\boldsymbol{u}), p$ ) (in Pa s) corresponding to the $\mu(I)$-rheology at different times during the granular mass collapse. The numerical resolution is $h=31.25 \times 10^{-3} \mathrm{~m}$ (coarse mesh) and the computational domain is $\Omega=(0,0.8) \times$ $(0,0.2)$, where unit of length is metre.

At the final stage, $t=1.06 \mathrm{~s}$, the profile of the granular mass is noticeably well predicted by our simulation. Note, however, that the experimental runout distance is underestimated by about $7.5 \%$. We will show below that the value of the viscosity $\eta_{\mathrm{s}}$ has an influence on the runout distance, which can be more accurately predicted when smaller values of $\eta_{0}$ are used. Note that the simulation in [13] found a runout distance overestimated by only $1.4 \%$ but the height of the mass at the back wall was $10 \%$ smaller though in the experiment the material in this zone remained static. The slope of the deposit as well as the overall distribution of the granular mass is more accurate in our simulation than in [13].

It is well known [30, that the level-set method, especially the reinitialization procedure, which is mandatory so that the level-set function remains signed distance, cannot predict the angle of the interface with walls for contact line problems. However, as shown on figure 3 , the front of the deposit at $t=1.06 \mathrm{~s}$ is found to be almost as thin as the experimental one. Note that, according to [30], we have adapted the high order ENO and WENO schemes at the walls by reducing their order of accuracy when mesh points are close to the boundaries so that no extrapolations at exterior ghost points are used. This allowed to stabilize the level-set solver. We infer that the implementation of the Coulomb friction law $(22)-(23)$ at the boundaries is essential in order to obtain a so thin interface close to the ground level. Indeed, the frontal part of the experimental deposit at the final time has only one glass bead depth, that is $0.7 \mathrm{~mm} \pm 0.1 \mathrm{~mm}$, over a horizontal distance of $1.5 \mathrm{~cm}(\approx 20$ glass beads). As shown on figure 3 , the result from [13, where a different numerical treatment of the interface is used, overestimates the thickness of the deposit at the front and on a larger area, that is for $x \geq 0.25$, resulting in a less accurate estimate of the slope of the deposit.

As in [13, the volume loss of the granular mass during the numerical simulation is below $0.84 \%$. Also shown on figure 3 is the Euclidean norm of the velocity field inside the granular mass during the collapse. This provides information on the flow dynamics. As it has been observed in experiments and in other numerical simulations [8, 48, 10, 12, 13, two zones in the granular mass can be identified during the collapse: a static one, corresponding to the basal deposit, which is growing with time and has no motion, overlain by a mobile one where glass beads are moving. The deposit shown in blue represents a large part of the granular mass. The motion occurs in a thin sublayer, underneath the surface down to the ground and behind the front, and in the early stage of the collapse, that is for time $t \in[0,0.42] \mathrm{s}$. At $t=0.42 \mathrm{~s}$, the front has reached the maximum runout distance. During the second half of the experiment, i.e. for $t \geq 0.42 \mathrm{~s}$, the deposit represents an increasing part of the whole granular mass until a stationary state is reached for $t$ near $1.06 \mathrm{~s}$ (i.e. deposition of the whole granular mass).

Contours of the basal deposit and of the moving layer are also well defined by using the strain-rate $|\boldsymbol{D}(\boldsymbol{u})|$ as discriminant quantity. Indeed, in viscoplastic fluids, plugs and dead zones correspond to areas where the strain-rate vanishes; both strain-rate and velocity vanish in dead zones. On the other hand, non-zero values of the strain-rate indicate motion with deformations. This is clearly visible on figure 4 , which shows most intense shear along the bottom wall at the flow head.

On figure 5 representing the pressure, spatial small-scale oscillations, similar to characteristic lines for hyperbolic problems, develop as soon as motion starts $(t=0.06 \mathrm{~s})$ and are persistent during the whole collapse. Such oscillations have also been identified by [14 and seem to be inherent to pressure dependent rheology, certainly related to the "ill-posedness" nature of the mathematical model. More precisely, it is well known that the DP-model is ill-posed [18, 19] in the sense that pressure oscillations are likely to appear 

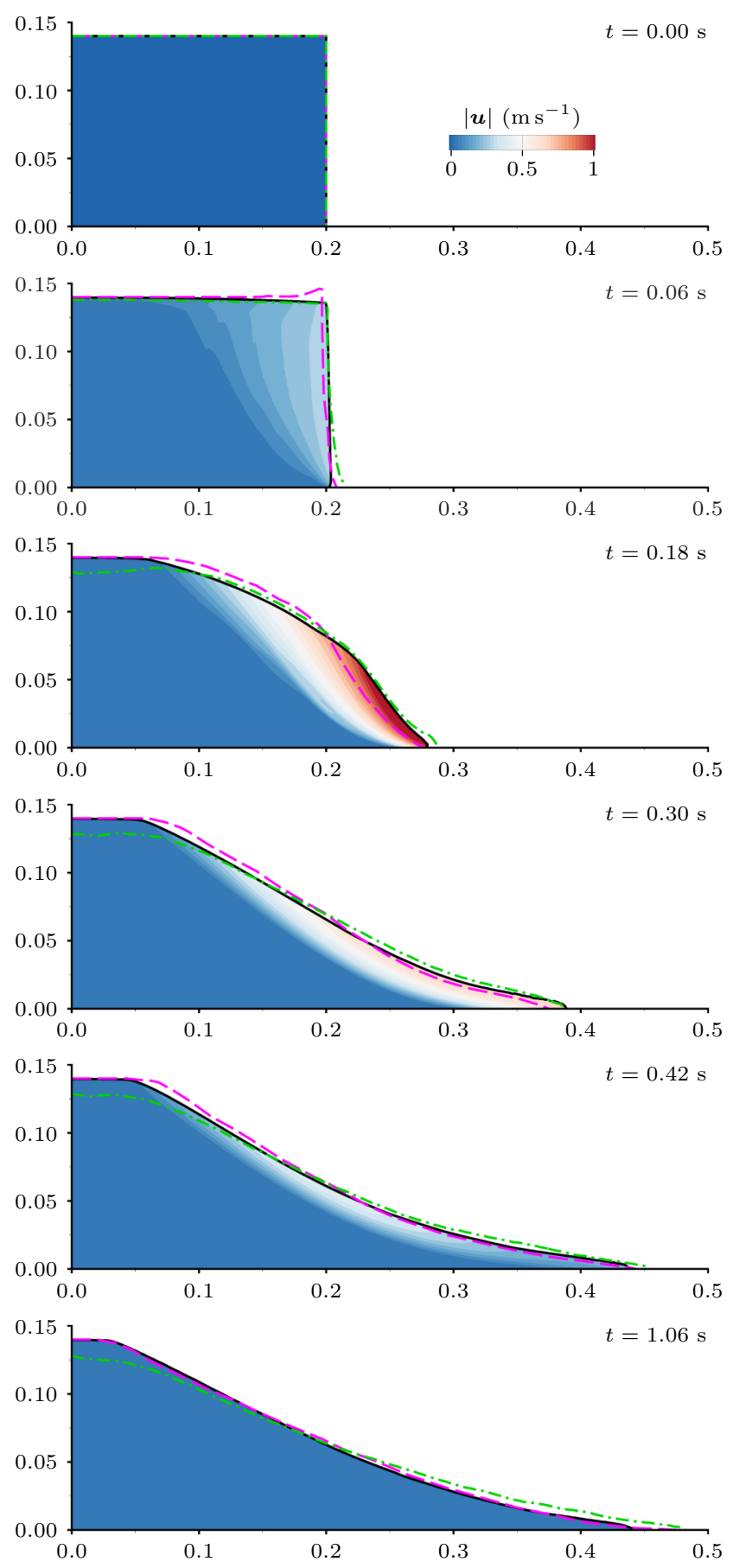

Figure 3: Comparison between numerical and experimental (pink dashed curve) results of the collapse of a granular mass. The color scale corresponds to the velocity Euclidean norm $|\boldsymbol{u}|$ (in $\mathrm{ms}^{-1}$ ) computed by using the DP-rheology. The black solid curve corresponds to the DP-rheology model while the green dot-dashed one is from [13. The numerical resolution is $h=0.78125 \times 10^{-3} \mathrm{~m}$ and the computational domain is $\Omega=(0,0.8) \times(0,0.2)$, where unit of length is metre. 

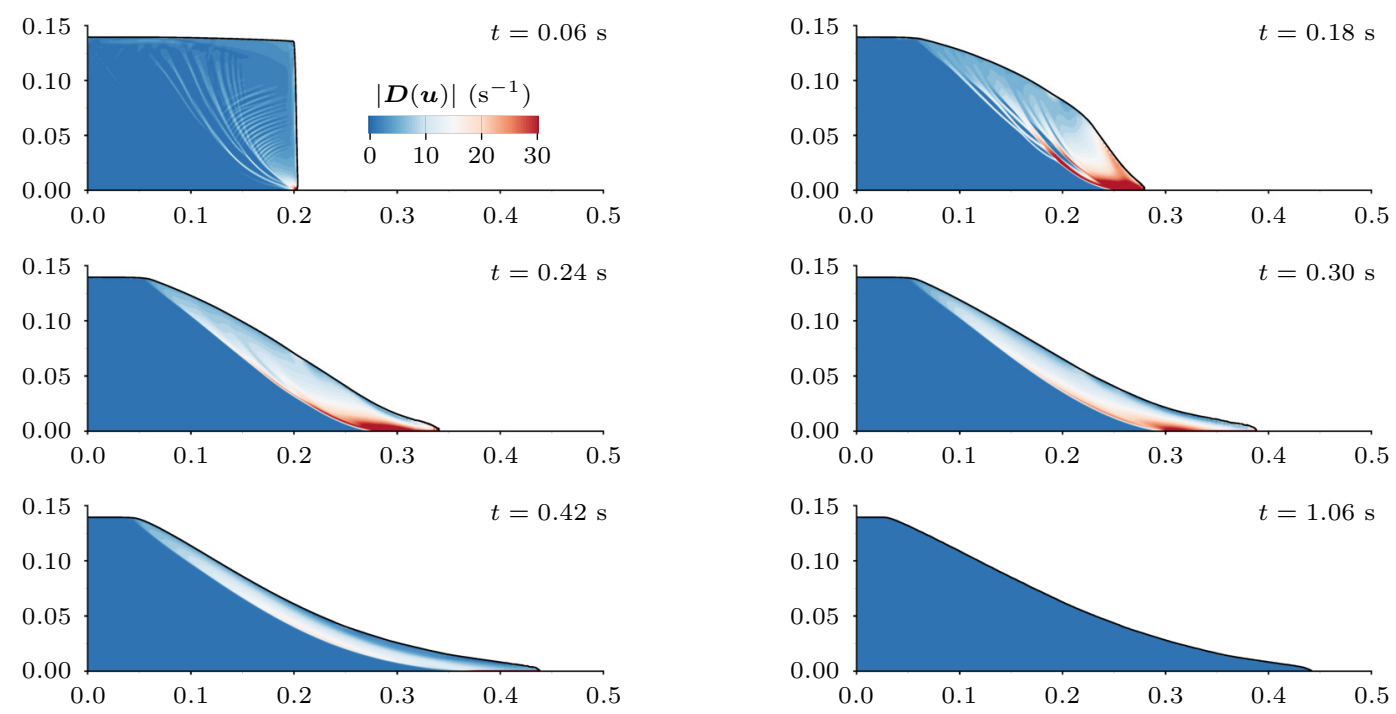

Figure 4: Strain-rate $|\boldsymbol{D}(\boldsymbol{u})|$ (in s ${ }^{-1}$ ) at different times during the granular mass collapse simulated with the DP-rheology. The numerical resolution is $h=0.78125 \times 10^{-3} \mathrm{~m}$ and the computational domain is $\Omega=(0,0.8) \times(0,0.2)$, where unit of length is metre.
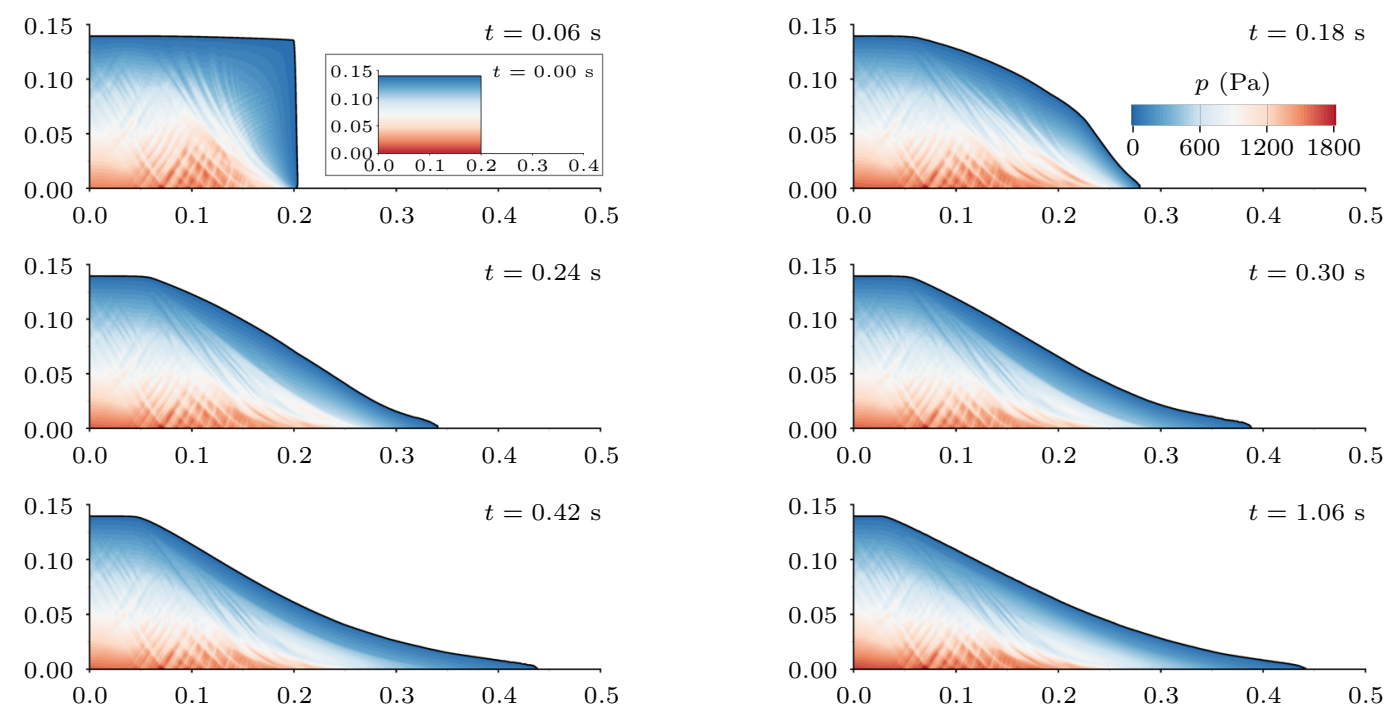

Figure 5: Pressure $p$ (in Pa) at different times of the granular collapse simulated with the DP-rheology. The initial static state at $t=0 \mathrm{~s}$ is also shown. Note that a different scale is used for the initial pressure for which maximum value is about $2129 \mathrm{~Pa}$. The numerical resolution is $h=0.78125 \times 10^{-3} \mathrm{~m}$ and the computational domain is $\Omega=(0,0.8) \times(0,0.2)$, where unit of length is metre. 


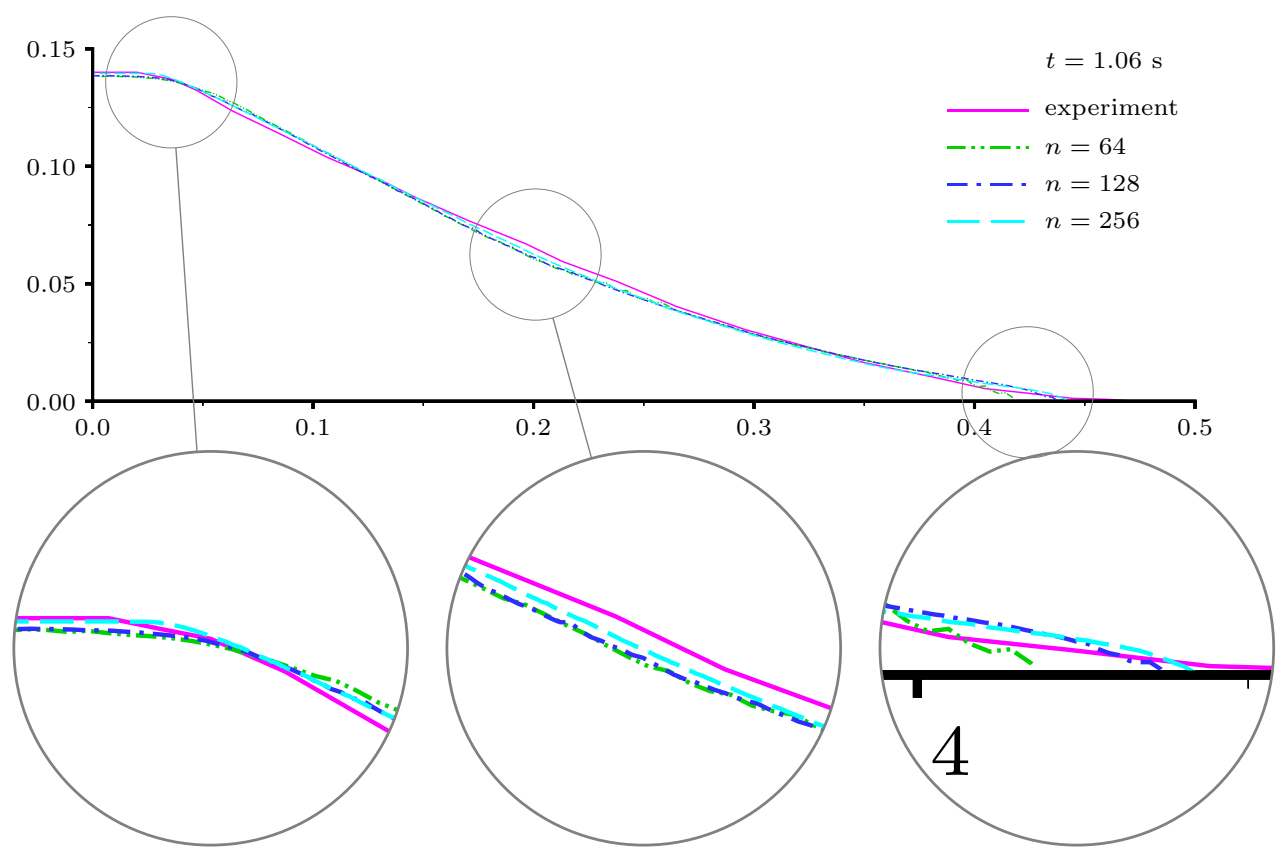

Figure 6: Profiles of the granular mass at the stationary state obtained with the DP-rheology with different mesh resolutions: $n=64\left(h=3.125 \times 10^{-3} \mathrm{~m}\right), n=128\left(h=1.5625 \times 10^{-3} \mathrm{~m}\right)$ and $n=256\left(h=7.8125 \times 10^{-4} \mathrm{~m}\right)$. Note that the size of the finer mesh is of the order of the particle diameter $(0.7 \pm 0.1 \mathrm{~mm})$.

and may grow so that solutions become unstable. Mathematically ill-posed problems suffer from unbounded growth of short wavelength perturbations, which necessarily lead to grid dependent numerical results that do not converge as the spatial resolution is enhanced. The $\mu(I)$-rheology, is well-posed for intermediate values of the inertial numbers $I$, but it remains ill-posed for both high and low inertial numbers [49]. Note that recently, in [50], changes to the functional form of the $\mu(I)$ curve have been considered in order to maximize the range of well-posed inertial numbers. [50] shows that when the inertial number is below a critical value there exists curves for which the equations are guaranteed to be well-posed. However, despite the presence of small-scale oscillations on pressure, the granular mass spreading is well predicted by the DP-model, and the shape of the granular mass as well as its contours are accurately computed. By refining the mesh, we did not observe amplifications of these oscillations and simulations remained stable. Moreover, pressure remains bounded and for all simulations $\max _{\Omega_{\mathrm{s}}} p \leq \rho_{\mathrm{s}} g H_{c} \approx 2129 \mathrm{~Pa}$, where $\Omega_{\mathrm{s}}$ is the part of the computational domain filled by glass beads $\left(\rho=\rho_{\mathrm{s}}\right)$.

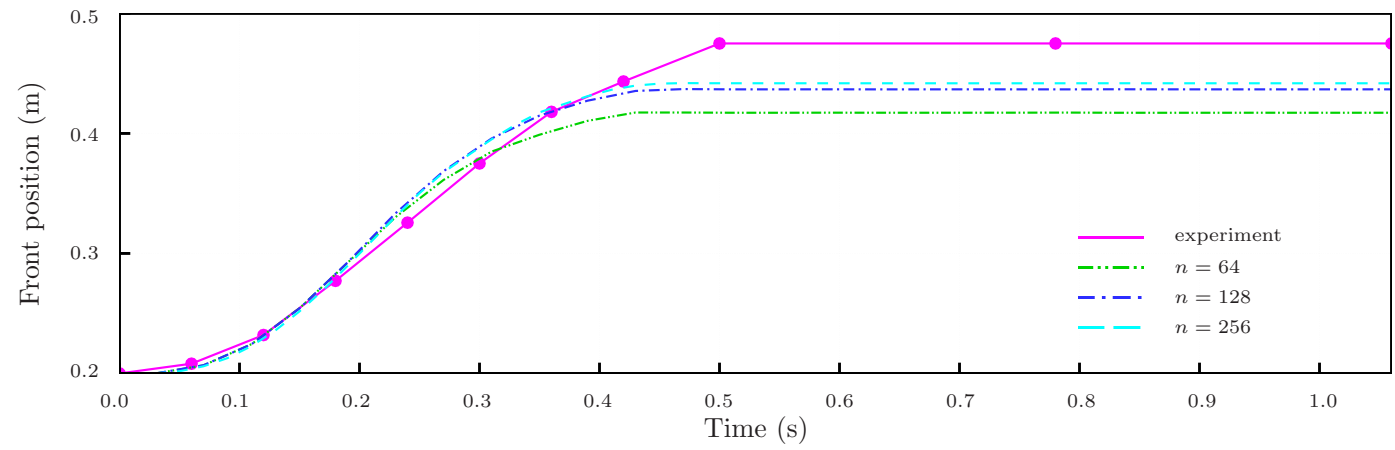

Figure 7: Plot of the front position as a function of time during the spreading of the granular mass. The front stops at $t=0.5 \mathrm{~s}$ while the upper part of the basal deposit still moves up to $t=1.06 \mathrm{~s}$ where a stationary state is reached. Results from simulations using different mesh sizes are shown and compared to the experimental measurement. Simulations in the computational domain $\Omega=(0,0.8) \times(0,0.2)$, where unit of length is metre, are performed with the DP-rheology. 

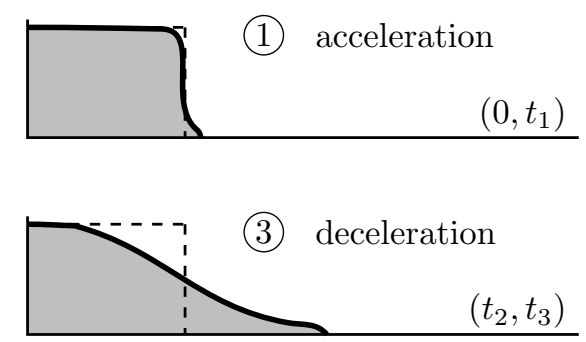
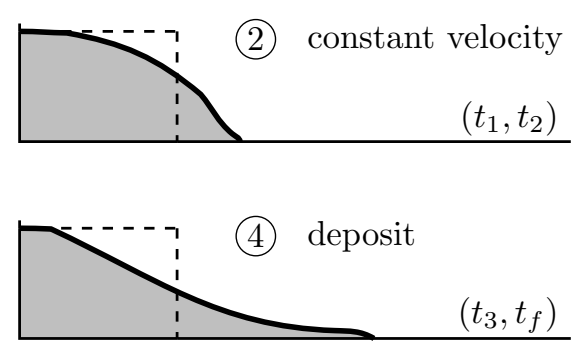

Figure 8: The four stages of the granular mass collapse.

\subsubsection{Numerical convergence}

In order to investigate the sensitivity of the numerical results with respect to the mesh resolution, we performed numerical simulations with $n=64,128$ and 256. The time step, adjusted in order to ensure CFL stability condition for the finer mesh, is equal to $\delta t=10^{-4} \mathrm{~s}$. The mesh size dependency is analyzed by focusing on the shape of the interface between the granular material and ambient air at $t=1.06 \mathrm{~s}$ (see figure 60, that is when the stationary state of the deposit is reached, and on the dynamics of the collapse by plotting the time evolution of the distance of the front at the bottom level from the back wall (see figure 7). It can be clearly observed on figure 6 that the deposit of the granular mass depends weakly on the resolution: results with different mesh sizes are very close to each other. The most important difference is found by comparing the computed runout distances: the value predicted with $n=64$ is smaller than values obtained with $n=128$ and $n=256$, which are close to each other, indicating numerical convergence. As it was mentioned in the previous section, the front of the simulated deposit is slightly thicker than in experiment. However, it is much thinner than what could be expected considering the fact that the level-set method is used to compute the interface. Indeed, the latter is known to have inherent difficulties to accurately compute the angle of the interface with walls for contact line problems such as the one considered in this paper. The use of the Coulomb friction law at the bottom boundary is compulsory in order to obtain a good approximation of the interface at the front end of the deposit. The simulation on the finer mesh was performed by using $32 \mathrm{MPI}$ processes during 3 days (waiting time). Note that the mesh size for $n=256$ is $h=0.78125 \times 10^{-3} \mathrm{~m}$, which corresponds to the diameter of the glass beads $(d=0.7 \pm 0.1 \mathrm{~mm})$.

A good agreement between simulated and experimental results is also obtained for the prediction of the mass spreading as shown on figure 7. Only the last measured point $(x=0.4750 \mathrm{~m})$ is missed by the numerical simulations, which stop at $0.442 \mathrm{~m}$ for the finest resolution. Note that on the last 1.5 centimeters, corresponding to 20 glass beads, the experimental deposit has a thickness of the order of $0.8 \mathrm{~mm}$, which means than the last visible particle locates at $x=0.4750 \mathrm{~m}$. For a numerical method solving a continuous fluid model with interface tracking, being able to capture a front end of one mesh size high extended on 20 mesh sizes seems to be unattainable. We will see in the next section that reducing the value of the viscosity $\eta_{0}$ improves the estimate of the runout distance. Despite this reasonably small discrepancy, the overall time history of the front is accurately captured. Indeed, as in the experiment, four time phases are clearly identified: the time interval $\left(0, t_{f}\right)$, where $t_{f}$ is the time where steady state is reached, can be split into four sub-intervals with bounds denoted by $t_{1}, t_{2}, t_{3}, t_{f}$ (see figure 8). During the first stage, for $t \in\left(0, t_{1}\right) \mathrm{s}$, acceleration occurs once the gate is lifted, then for $t \in\left(t_{1}, t_{2}\right) \mathrm{s}$ the front moves forward at a constant speed. In the following phase $t \in\left(t_{2}, t_{3}\right) \mathrm{s}$ the flow decelerates and, finally, over a longer time interval $t \in\left[t_{3}, t_{f}\right] \mathrm{s}$ the front has reached the runout distance and does not advance anymore, while the upper surface of the basal deposit still moves upwards very slowly until it reaches the surface of the granular mass at $t=t_{f}=1.06 \mathrm{~s}$. From figure 7 , we may infer values of the bounds $t_{i}, i=1,2,3: t_{1} \approx 0.1 \mathrm{~s}, t_{2} \approx 0.35$ $\mathrm{s}$ and $t_{3} \approx 0.45 \mathrm{~s}$. Note that during the last time interval, $t \in(0.45,1.06) \mathrm{s}$, which is more than half of the whole time interval of the simulation, the motion at the surface of the granular material is very slow while the front has already reached the runout distance. During the constant propagation speed phase the front is found in the experiment to move at a speed equal to $0.775 \mathrm{~m} \mathrm{~s}^{-1}$ while the numerical simulations predict a faster displacement $\approx 0.952 \mathrm{~m} \mathrm{~s}^{-1}$. This was also observed in 13 .

An important parameter in the numerical algorithm is the pseudo-relaxation coefficient $\theta$ added in the Bingham projection in order to ensure geometrical convergence of the Picard's algorithm used to solve the coupling in (31) (see also [23, 24]). This parameter must be chosen sufficiently small in order to ensure that the plastic part of the stress tensor is well resolved. The theory suggests that $\theta$ should be of the order of the time step $\delta t$ (see [23, 24]) ensuring the time scheme to be first order accurate. In practice, we found that 


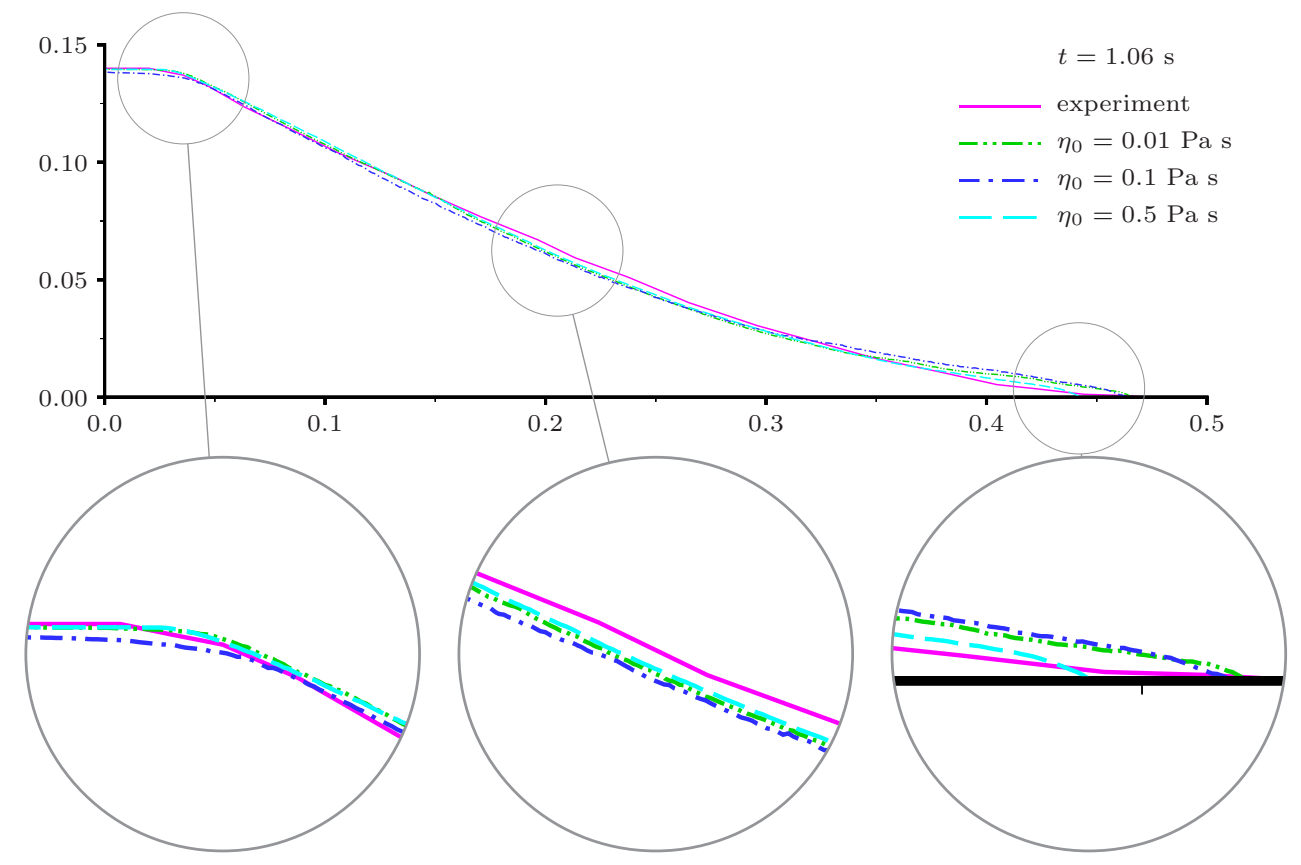

Figure 9: Profile of the granular deposit at $t=1.06 \mathrm{~s}$ obtained for various values of the viscosity $\eta_{0}$ in the DP-rheology. The resolution corresponds to the finer mesh with $h=0.78125 \times 10^{-3} \mathrm{~m}$ and the computational domain is $\Omega=(0,0.8) \times(0,0.2)$, where unit of length is metre.

using $\theta=10 \delta t$ provided accurate predictions of the slump dynamics. This value is optimal in the sense that it reduces the computational cost and ensures accurate results.

\subsubsection{Influence of the value of the viscosity $\eta_{0}$ on the numerical results}

We conclude the analysis of the results obtained with the DP-model for the 0.7 aspect ratio column by estimating the influence of the viscosity coefficient $\eta_{0}$ on the results. As it was mentioned in section 4.1.2, all previously discussed simulations were conducted with $\eta_{0}=0.5 \mathrm{~Pa}$ s. Using smaller values of $\eta_{0}$ will also attest the robustness of our code and of the implementation with a bi-projection scheme in the framework of level-set method of the DP-model. Investigating the dependency of the results upon this parameter is relevant. Indeed, if the main characteristics of the collapse of granular columns are found to be almost insensitive to the value of the viscosity, then the use of a constant viscosity model is suitable as it avoids the numerical difficulties of a spatio-temporal variable viscosity as in the $\mu(I)-$ rheology. Therefore, in order to complete our study, we performed simulations with $\eta_{0}=0.1 \mathrm{~Pa}$ s and $0.01 \mathrm{~Pa}$ s. Note that in [13, values of the viscosity in the range $[0.1,10] \mathrm{Pa}$ s for the DP-model, but with a different numerical implementation, have been used. The authors mentioned that, for computational issues, they were not able to take smaller values. Setting $\eta_{0}=0.01 \mathrm{~Pa} \mathrm{~s}$ with our parallel code did not induce any technical difficulty. The time step, for stability reason, was divided by two and the number of MPI processes was doubled in order to keep constant the computational waiting time.

Figure 9 shows profiles of the granular mass at stationary state obtained with the considered values of the viscosity and compared with the experimental one. First of all, the overall distribution of the granular mass in the domain is weakly influenced by the value of the viscosity. The best result is obtained with $\eta_{0}=0.01$ $\mathrm{Pa} \mathrm{s}$ for which an almost perfect match with the experimental deposit is found. Indeed, the runout distance increases as $\eta_{0}$ decreases and almost matches the experimental value. Figure 10 indicates a convergence with respect to the value of $\eta_{0}$. The height of the granular mass at the back wall is also well approximated: $0.1384 \mathrm{~m}$, which is only $1.5 \%$ below the experimental value. All profiles are close to each other. The front of the granular mass propagates slightly faster for values of the viscosity smaller than $0.5 \mathrm{~Pa}$ s but the curves for $\eta_{0}=0.1 \mathrm{~Pa}$ s and $0.01 \mathrm{~Pa}$ s are close to each other up to $t=0.3 \mathrm{~s}$ (see figure 10). Figure 10 is completed by figure 11, which shows profiles at different times during the collapse obtained with $\eta_{0}=0.1 \mathrm{~Pa} \mathrm{~s}$ and $\eta_{0}=0.01 \mathrm{~Pa}$ s. Differences in the profiles occur in the area $x \in[0.05,0.1] \mathrm{m}$ where the height of the granular flow is larger with $\eta_{0}=0.01 \mathrm{~Pa} \mathrm{~s}$. As a consequence, the slopes of the profiles are steeper for $x \in[0.1,0.2]$ $\mathrm{m}$. The overall behavior of the collapse is well captured and values of the viscosity have minor effects on the 


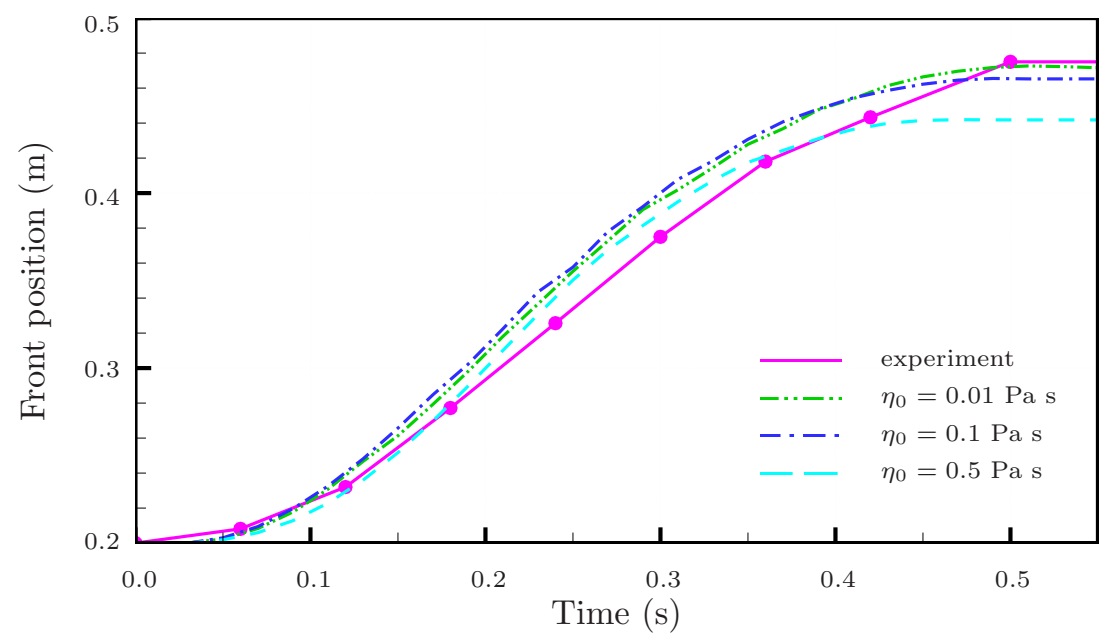

Figure 10: Plot of the front position as a function of time during the spreading of the granular mass. The front stops at $t=0.5 \mathrm{~s}$. Results from simulations performed with the DP-rheology and using different values of the viscosity $\eta_{0}$ are shown and compared to the experimental measurement. The computational domain is $\Omega=(0,0.8) \times(0,0.2)$, where unit of length is metre, and the mesh size is $h=0.78125 \times 10^{-3} \mathrm{~m}$.
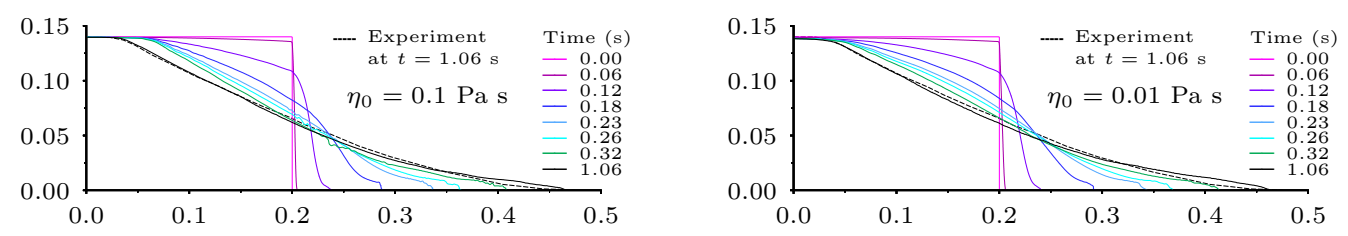

Figure 11: Time evolution of the profile of the granular mass during its collapse obtained with the DP-rheology and simulated with viscosity $\eta_{0}=0.1 \mathrm{~Pa} \mathrm{~s}$ (left) and $\eta_{0}=0.01 \mathrm{~Pa} \mathrm{~s}$ (right). The computational domain is $\Omega=(0,0.8) \times(0,0.2)$, where unit of length is metre, and the mesh size is $h=0.78125 \times 10^{-3} \mathrm{~m}$.

flow dynamics.

On figure 12 , we observe that the dynamic pressure is affected by the value of the viscosity. Namely, in the area corresponding to the basal deposit, the pressure has less small-scale oscillations for the simulations performed with the values $\eta_{0}=0.1$ and $0.01 \mathrm{~Pa} \mathrm{~s}$ than with $\eta_{0}=0.5 \mathrm{~Pa} \mathrm{~s}$. These oscillations are persistent and still visible but mainly in the sublayer where the flow motion occurs.

As a summary, the dependency of the numerical results upon the value of $\eta_{0}$ is very weak, thus attesting the robustness of both the DP-rheology with constant viscosity and the numerical implementation used in this paper. For this type of granular flow, it appears that the main contribution to the effective viscosity is induced by the plasticity criterion, namely the term $\tan (\alpha) p /|\boldsymbol{D}(\boldsymbol{u})|$ in regions where $\boldsymbol{D}(\boldsymbol{u}) \neq 0$. Therefore, the contribution due to the spatio-temporal variable viscosity $\eta(\boldsymbol{D}(\boldsymbol{u}), p)$ is negligible. However, a small but non-zero constant viscosity helps to maintain the cost of the numerical simulations at a reasonable level. Indeed, the time step had to be reduced for the value $\eta_{0}=10^{-3} \mathrm{~Pa} \mathrm{~s}$. The flow dynamics for the collapse of dense and dry granular columns is mainly controlled by the Drucker-Prager plasticity criterion (4) with friction coefficient $\mu=\tan (\alpha)$.

\subsubsection{The HP-rheology}

We now present simulations performed with the HP-model defined by $14-15$ and characterized by a yield stress proportional to the hydrostatic pressure. Unlike the DP-rheology, the HP-model is well-posed (see [21]). However, from the best of our knowledge, the HP-model has never been used to simulate the gravitational collapse of a granular column. We hereafter investigate its applicability in this context.

As shown on figure 13, the prediction, provided by the HP-rheology, of the shape of the granular deposit at steady state is acceptable: the height at the back wall has the correct value, the slope of the deposit downstream the left plateau is slightly larger than the experimental one, and the runout distance is underestimated by $24 \%$. However, the HP-model fails to reproduce the correct dynamics. At $t=0.18 \mathrm{~s}$, a small bump appears at the right upper corner of the granular mass and further develops into a growing, non physically relevant protuberance up to $t=0.42 \mathrm{~s}$. The strength of the threshold, vertically stratified by 

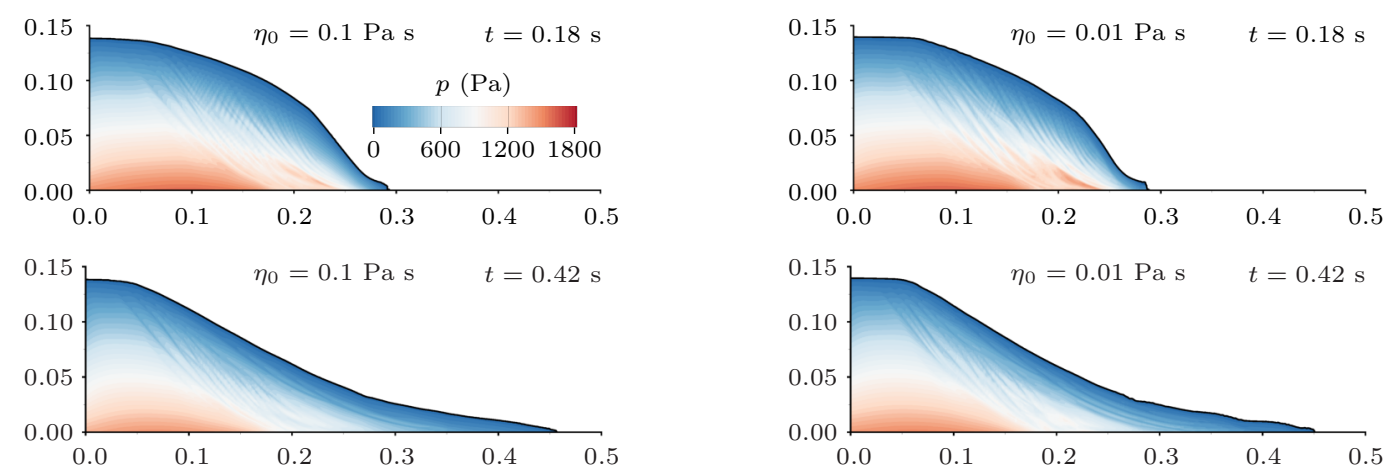

Figure 12: Comparison of dynamic pressure $p$ (in $\mathrm{Pa}$ ) with respect to different viscosity values $\eta_{0}$ (in $\mathrm{Pa} \mathrm{s}$ ). The granular collapse is simulated with the DP-rheology. The numerical resolution is $h=0.78125 \times 10^{-3} \mathrm{~m}$ and the computational domain is $\Omega=(0,0.8) \times(0,0.2)$, where unit of length is metre.
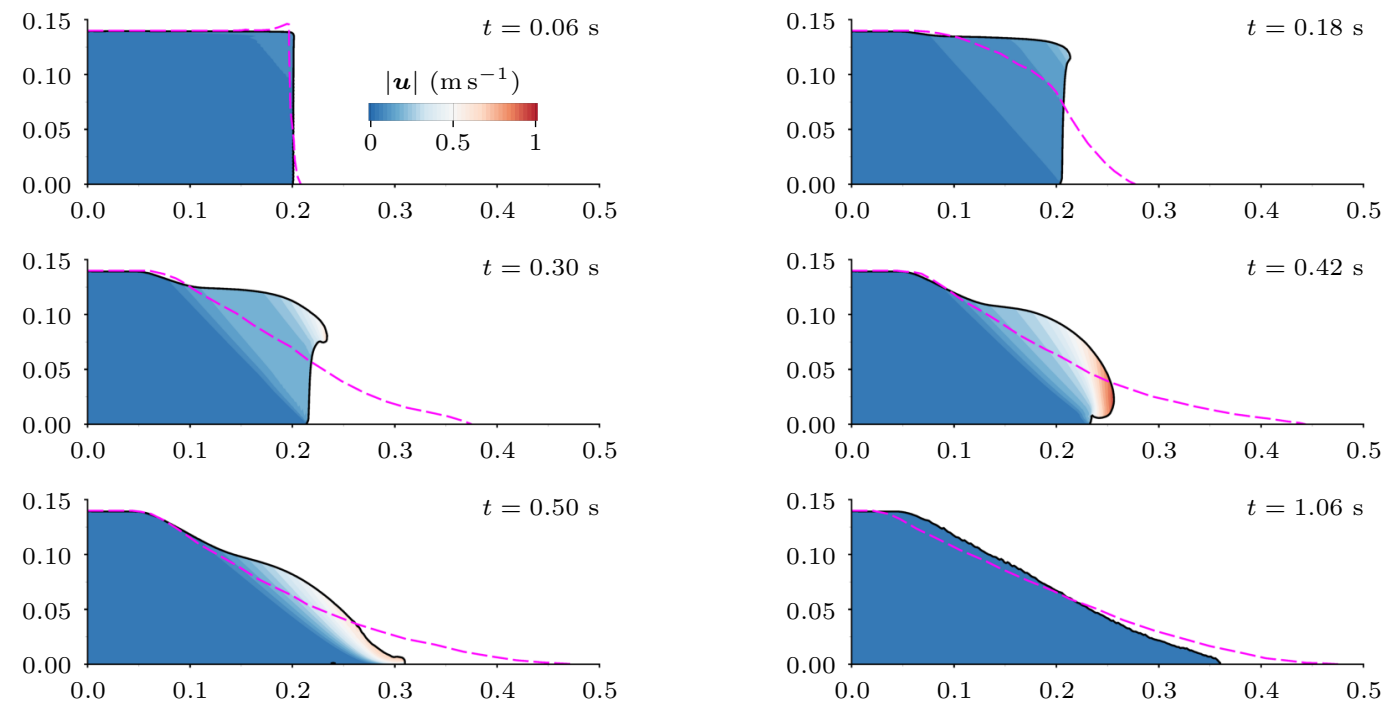

Figure 13: Comparison between numerical (black solid curve) and experimental (pink dashed curve) results of the collapse of a granular mass. The color scale corresponds to the velocity Euclidean norm $|\boldsymbol{u}|\left(\right.$ in $\left.\mathrm{m} \mathrm{s}^{-1}\right)$. The HP-rheology model is used. The numerical resolution is $h=0.15625 \times 10^{-2} \mathrm{~m}$ and the computational domain is $\Omega=(0,0.8) \times(0,0.2)$, where unit of length is metre. The viscosity is constant : $\eta_{0}=0.5 \mathrm{~Pa} \mathrm{~s}$.
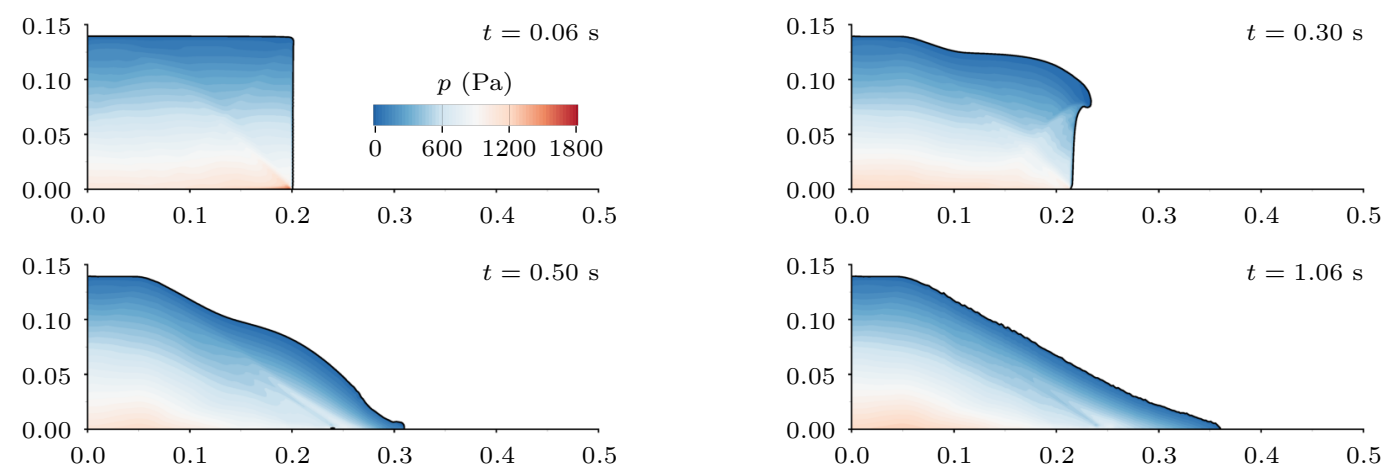

Figure 14: Pressure $p$ (in $\mathrm{Pa}$ ) at different times during the granular mass collapse computed with the HP-rheology. The numerical resolution is $h=0.15625 \times 10^{-2} \mathrm{~m}$ and the computational domain is $\Omega=(0,0.8) \times(0,0.2)$, where unit of length is metre. The viscosity is constant : $\eta_{0}=0.5 \mathrm{~Pa} \mathrm{~s}$. 
definition, is far too strong at the ground level so that the right margin of the base of the granular column moves very slowly as a block and the interface with air remains nearly vertical. Capturing the roll-up of the interface and the fall of the nose-like bump on the horizontal plane, near $t=0.42 \mathrm{~s}$, is critical and difficult for an interface tracking method. This demonstrates the robustness of our implementation of the level set method to simulate viscoplastic dambreak problems. As in the experiment, a stationary state is reached for $t \approx 1.06 \mathrm{~s}$. As expected, due to the well-posedness of the HP-model, the pressure gradient is smooth and has no oscillations (see figure 14). In summary, the HP-model produces an acceptable steady state (i.e. deposit) but fails to reproduce the dynamics of the granular slump.

\subsection{Collapse of a dry granular column with aspect ratio 2 over a smooth surface}

\subsubsection{Experimental setup}

Note that from the best of our knowledge, the results presented below are the first ones comparing numerical simulations with an experiment carried out by [20] with an initial column of width $L_{c}=20 \mathrm{~cm}$ and height $H_{c}=40 \mathrm{~cm}$. Moreover, due to the fact that the aspect ratio of the column is larger than unity, depth-averaged models can not be used. The glass beads used in this case are ten times finer than the ones used in the previous experiment and have a (mean) diameter $d=0.08 \mathrm{~mm}$. The reservoir $\left(0, L_{c}\right) \times\left(0, H_{c}\right)$ is connected with a channel with a smooth base, which is $10 \mathrm{~cm}$ wide and $3 \mathrm{~m}$ long, through a gate opened at $t=0 \mathrm{~s}$. In our numerical simulations, the gate is not modeled and we assume that the whole granular column is released at $t=0 \mathrm{~s}$. The particle density is $\rho_{\mathrm{p}}=2500 \mathrm{~kg} \mathrm{~m}^{-3}$, which leads to an apparent flow density $\rho_{\mathrm{s}}=1450 \mathrm{~kg} \mathrm{~m}^{-3}$ (a mass volume fraction of 0.58 is taken into account). The static (inner) friction coefficient for the considered glass beads is $27^{\circ}$, which is equal to the material repose angle 3 .

\subsubsection{Influence of the basal friction angle}

The friction angle of the glass beads on the plexiglass plates of the channel is estimated by considering, at increasing slope angles, onset of motion of a layer of beads overlain by a solid metal block. When the beads are free to roll, the block moves at an angle of $8^{\circ} \pm 1^{\circ}$. Note that with this technique, it is almost impossible to be sure that glass beads do not overlap and that the thickness of the basal layer remains equal to one particle diameter during the test. Furthermore, the apparent low friction angle is most certainly due to rolling of the particles. In contrast, when the layer of glass beads is glued to the block, the onset of motion of the block occurs at an angle of $19^{\circ}$, which is assumed to be close to the friction angle between the beads and the plates. Notice that this value was considered in [7. The actual friction angle between the beads and the plates is likely to be close but smaller than $19^{\circ}$, owing to some rolling component, as considered hereafter. Unlike for the experiment at aspect ratio 0.7, we assume that the friction angles for all contact surfaces, namely both the vertical back wall and the bottom plate, have the same value, which is chosen close to $19^{\circ}$ as stated above. In order to estimate the influence of the basal friction angle on the dynamics of the granular mass slump, we retained the three following representative values: $12.5^{\circ}, 15^{\circ}$ and $17.5^{\circ}$. On figure 15. we compare the mass profiles at the final time, namely $t=1.36 \mathrm{~s}$, of the collapse of the granular column obtained with the DP-rheology, on a grid with $768 \times 256$ mesh points ( $h=0.46 / 256 \approx 1.8 \mathrm{~mm}$ ); the time step has to be set to $\delta t=10^{-4} \mathrm{~s}$ in order to ensure numerical stability. Note that in this case the mesh size $h$ is 22.5 times larger than the particle size. Using a mesh with two times more points will increase the amount of computational resources (CPU time and number of MPI processes) required but remains feasible. Nevertheless, using a mesh as fine as the glass beads as in section 4.1 is out of reach. Therefore, the choice of a grid with $768 \times 256$ mesh points is a good compromise between the amount of computational resources involved and the waiting time to get simulations done (6 days with 32 MPI processes). The value of the viscosity, $\eta_{0}=0.1 \mathrm{~Pa} \mathrm{~s}$, was retained. By performing a simulation on a coarser grid $(192 \times 64$ mesh points $)$ with the $\mu(I)$-rheology, we found that $\eta(|\boldsymbol{D} \boldsymbol{u}|, p)$ is bounded from above by $0.2 \mathrm{~Pa}$ s and in a narrow layer at the top of the granular mass, during the collapse, $\eta(|\boldsymbol{D} \boldsymbol{u}|, p)$ takes values smaller than 0.1 Pa s. By assuming that, in the static part of the granular mass, plasticity due to the Drucker-Prager dominates over viscous effects (as discussed in section 4.1.2), we chose $0.1 \mathrm{~Pa}$ s for the constant viscosity. As shown above at low aspect ratio of 0.7 , we will further show that no significant changes are found when the value of $\eta_{0}$ is decreased.

Figure 15 shows that the values $15^{\circ}$ and $17.5^{\circ}$ for the basal friction angle give very similar approximations of the profile of the final deposit, i.e. at $t=1.36 \mathrm{~s}$. For these coefficients, the runout distances are respectively underestimated by $5.9 \%$ and $15 \%$. Close to the back wall, i.e. for $x \leq 0.3 \mathrm{~m}$, the numerical profiles, obtained with $\tan \left(15^{\circ}\right)$ and $\tan \left(17.5^{\circ}\right)$, are respectively $5.5 \%$ and $2.85 \%$ below the experimental one. With the smallest friction angle $12.5^{\circ}$, the front stops at $1.282 \mathrm{~m}$ compared to $1.213 \mathrm{~m}$ for the experiment but the height of the granular mass on the interval $x \in[0,0.35] \mathrm{m}$ is underestimated by $11.3 \%$. 


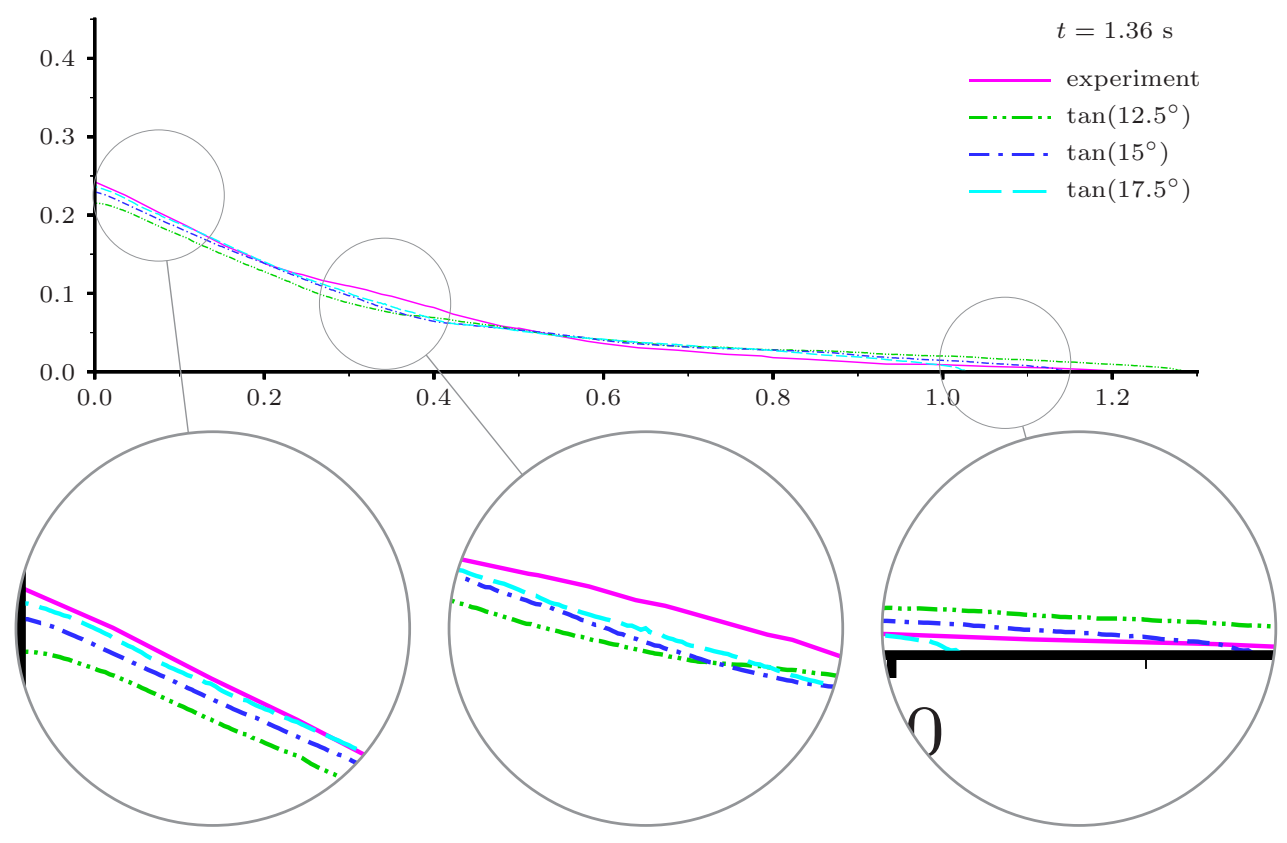

Figure 15: Profiles of the granular mass at the final time of the mass spreading obtained with the DP-rheology for the $(0,0.2) \times(0,0.4)$ initial column, where unit of length is metre. Profiles obtained with different basal friction angles $\left(12.5^{\circ}, 15^{\circ}\right.$ and $17.5^{\circ}$ ) are shown and compared with the experimental result.

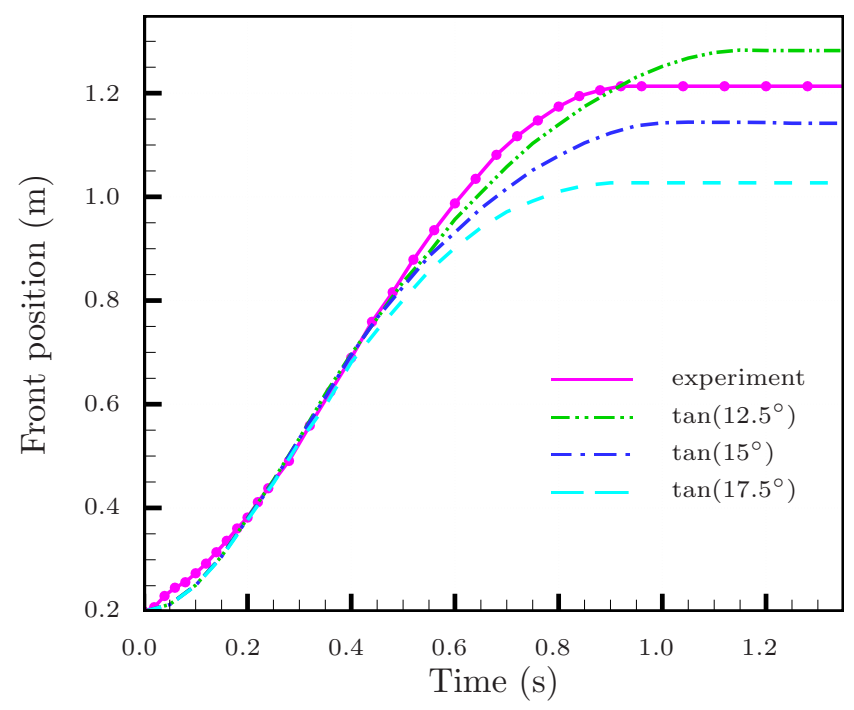

Figure 16: Time evolution of the front position during the spreading of the granular mass. The front stops at $t=0.9 \mathrm{~s}$ and the upper part of the basal deposit grows up to $t=1.36 \mathrm{~s}$ where a steady state is reached. Results obtained with three different values of the basal friction angle $\left(12.5^{\circ}, 15^{\circ}\right.$ and $\left.17.5^{\circ}\right)$ are shown and compared with the experimental result. 
The simulation with $\tan \left(15^{\circ}\right)$ matches fairly well the experiment, which corresponds to a particle-wall friction coefficient in between the two experimental values of $8^{\circ}$ and $19^{\circ}$. In experiments, however, friction occurs also at the lateral walls. The effect of the lateral walls is probably small because the height of the flowing layer becomes increasingly small compared to the channel width when the granular mass spreads, but it is certainly non-negligible. If lateral friction was considered in the simulations then at given friction coefficient the runout distance, for instance, would be smaller than observed in the present simulations. In consequence, lower coefficients would be required to match the experiment. As discussed above, a possible explanation is that the particles at the flow base in the experiment may have some rolling component whereas the particles glued at the base of the solid block used for the test are truly static.

Note that for this initial column, the final deposit clearly has two slopes: a first one of $7^{\circ}$ for $x \geq 0.5$ $\mathrm{m}$ up to the front of the granular mass and a steeper one of $29^{\circ}$ for $x \leq 0.35 \mathrm{~m}$. These slopes are well approximated by all simulations (see figure 15). As for the previous experiment, the level-set method is able to predict a thin deposit near the front: the angle between the surface of the deposit and the flat horizontal plate is very small. Indeed, we respectively find $3^{\circ}, 4.85^{\circ}$ and $10^{\circ}$ for increasing values of the basal friction compared to $2.5^{\circ}$ for the experiment. As a summary, dependence of the results upon the basal friction, for the values considered here, is weak and the three different results are more than acceptable when compared to the experiment.

By looking at the time evolution of the front of the granular mass during the collapse shown on figure 16 . we deduce as in section 4.1.2 that the dynamics of the slump can be divided in four phases: a short acceleration phase followed by a time period where the propagation speed of the front is constant, then a deceleration phase and finally a long time period with a very slow motion until the flow stops. Due to the fact that effects of the gate is not accounted for in our simulations, a small time delay in the sliding of the front is visible during the acceleration period, that is for $t \in[0,0.15] \mathrm{s}$. In the second phase, that is $t \in[0.15,0.5]$ $\mathrm{s}$, all the three basal friction coefficients provide curves that can not be differentiated and are superposed with the experimental one until the deceleration phase. The propagation speed is here better approximated than for the shorter column shown on figure 7. However, simulations for the different values of the basal friction coefficient have slightly different deceleration phases as they reach different runout distances.

\subsubsection{Numerical results obtained with $15^{\circ}$ as basal friction angle: comparison with experiment.}

According to the previous discussion, the basal friction coefficient is chosen equal to $\kappa_{\mathrm{b}}=\tan \left(15^{\circ}\right)$ for further analysis. On figure 17, profiles of the granular mass computed with the DP-model are shown at different times during the collapse and compared with the experimental one. Except during the acceleration phase, i.e. for $t \leq 0.16 \mathrm{~s}$, where the experimental front is further advanced than the numerical one, the numerical profiles are in good agreement with the experimental ones. During the deceleration phase $(t \geq 0.4$ s), the granular mass consists of two areas with different surface slopes: a bend on the interface occurs at $x \approx 0.4 \mathrm{~m}$.

At the final (stationary) time $t=1.36 \mathrm{~s}$, the slope of the interface at the back wall is particularly well approximated by the numerical simulation. In the ranges $x \in[0,0.3] \mathrm{m}$ and $x \in[0.5,1.1] \mathrm{m}$, both profiles are almost identical while the profile predicted by the numerical simulation is slightly below the experimental curve for $x \in[0.3,0.5] \mathrm{m}$. By looking at the norm of the velocity field $|\boldsymbol{u}|$ (in $\mathrm{m} \mathrm{s}^{-1}$ ) (in figure 17) and the strain-rate $|\boldsymbol{D}(\boldsymbol{u})|\left(\right.$ in s $^{-1}$ ) (in figure 19), a basal deposit growing with time is clearly visible. Also, as already observed for the 0.7 aspect ratio column, the motion takes place in a thin surface layer. The velocity takes its largest values in a narrow zone behind the front. The maximum velocity at the front is of the order of $1.6 \mathrm{~m} \mathrm{~s}^{-1}$.

Both the pressure (figure 18) and the strain-rate (figure 19) exhibit short wave oscillations during the whole collapse. As this was the case for the 0.7 aspect ratio column, despite these oscillations the simulations remain stable and the pressure is bounded by $\rho_{\mathrm{s}} g H_{c} \approx 6082 \mathrm{~Pa}$. As it was discussed in section 4.1.2, pressure oscillations are well-known for the DP-rheology and are related to the ill-posedness of the model. Nevertheless, the DP-model permits us to obtain accurate simulations of the collapse of granular columns.

\subsubsection{Influence of the value of the viscosity $\eta_{0}$ on the numerical results}

As for the 0.7 aspect ratio column (section 4.1.4), we investigate the influence of the constant viscosity $\eta_{0}$ on the numerical results. Therefore, we performed a numerical simulation with $\eta_{0}=10^{-2} \mathrm{~Pa}$ s, which is 20 times smaller than the value predicted by the $\mu(I)$-rheology for this configuration (see section 4.2.2). The time step $\delta t$ was set to $5 \times 10^{-5} \mathrm{~s}$ in order to ensure stability (CFL criterion) and 64 MPI processes were used. Decreasing further the value of the viscosity as in the 0.7 aspect ratio column is not feazible here, as this will require to take a smaller time step: $\delta t \approx \times 10^{-5} \mathrm{~s}$ for $\eta_{0}=10^{-3} \mathrm{~Pa}$ s. Indeed, a taller column 

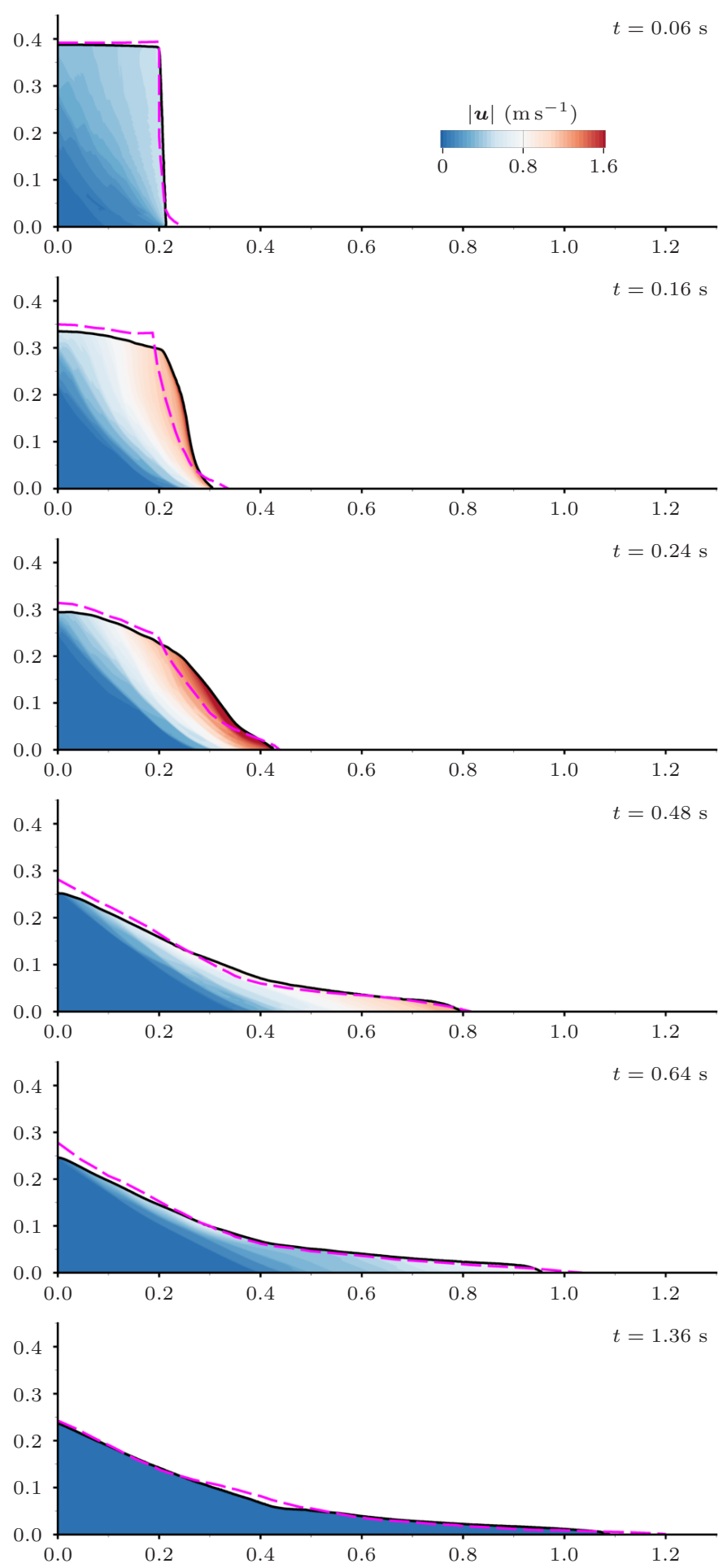

Figure 17: Comparison between numerical (black solid curve) and experimental (pink dashed curve) results of the collapse of a granular mass. The color scale corresponds to the velocity Euclidean norm $|\boldsymbol{u}|$ (in $\mathrm{m} \mathrm{s}^{-1}$ ) computed by using the DP-rheology. The numerical resolution is $h=1.796875 \times 10^{-3} \mathrm{~m}(n=256)$, the computational domain is $\Omega=(0,1.38) \times(0,0.46)$, where unit of length is metre, and the basal friction angle is $\alpha_{\mathrm{b}}=15^{\circ}$. 

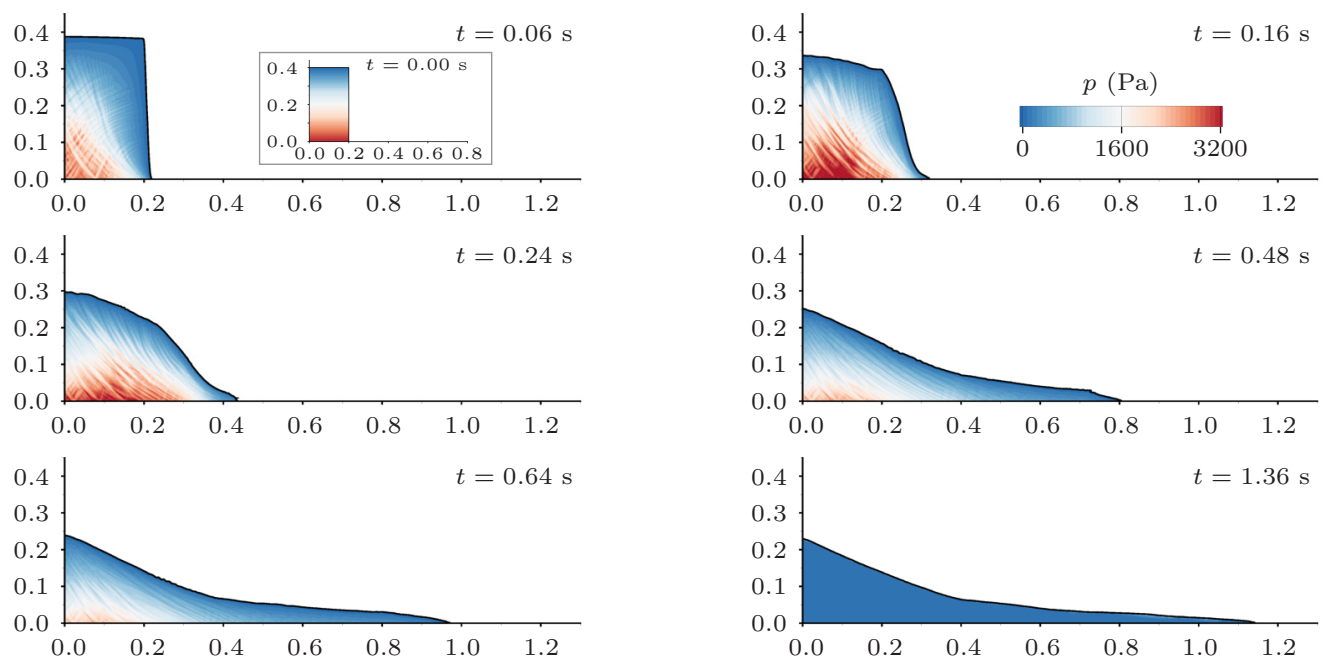

Figure 18: Pressure $p$ (in Pa) at different times during the granular mass slump simulated with the DP-rheology. The initial static state at $t=0 \mathrm{~s}$ is also shown. Note that a different scale is used for the initial pressure, which takes a larger maximum value $(\approx 6082 \mathrm{~Pa})$. The numerical resolution is $h=1.796875 \times 10^{-3} \mathrm{~m}(n=256)$, the computational domain is $\Omega=(0,1.38) \times(0,0.46)$, where unit of length is metre, and the basal friction angle is $\alpha_{\mathrm{b}}=15^{\circ}$.
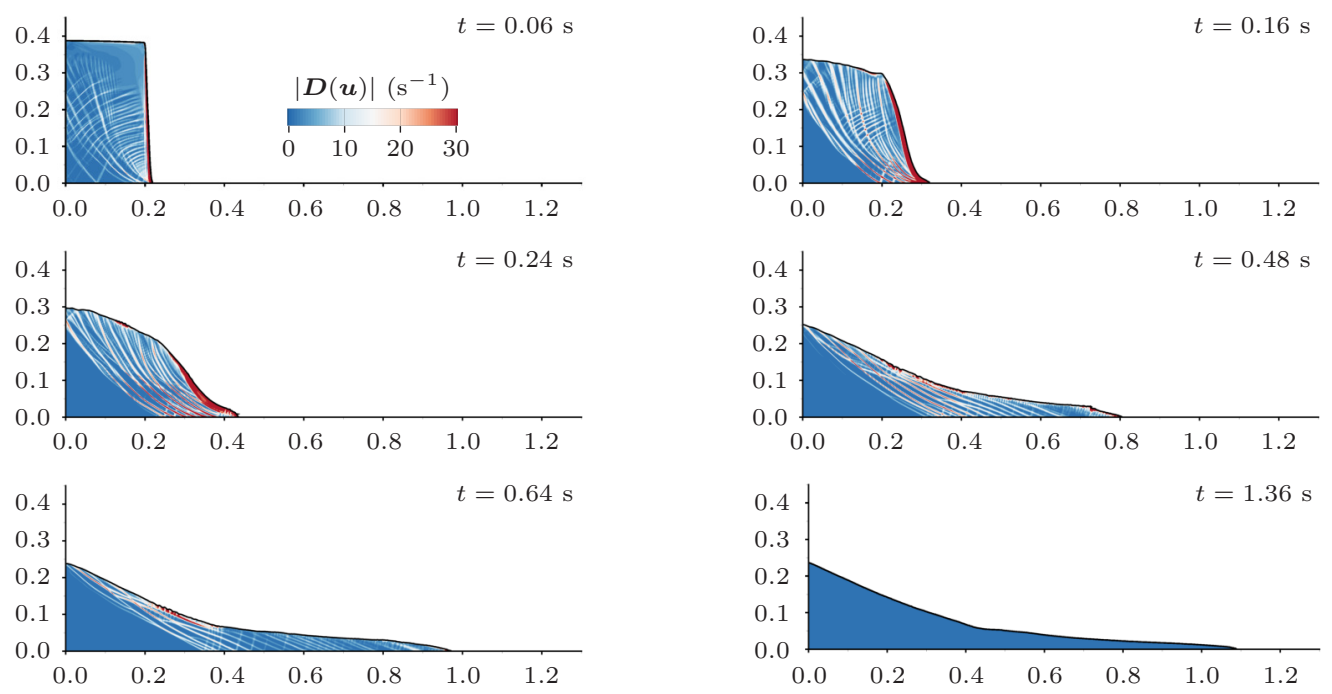

Figure 19: Strain-rate $|\boldsymbol{D}(\boldsymbol{u})|$ (in s ${ }^{-1}$ ) at different times during the granular mass slump simulated with the DP-rheology. The numerical resolution is $h=1.796875 \times 10^{-3} \mathrm{~m}(n=256)$, the computational domain is $\Omega=(0,1.38) \times(0,0.46)$, where unit of length is metre, and the basal friction angle is $\alpha_{\mathrm{b}}=15^{\circ}$. 


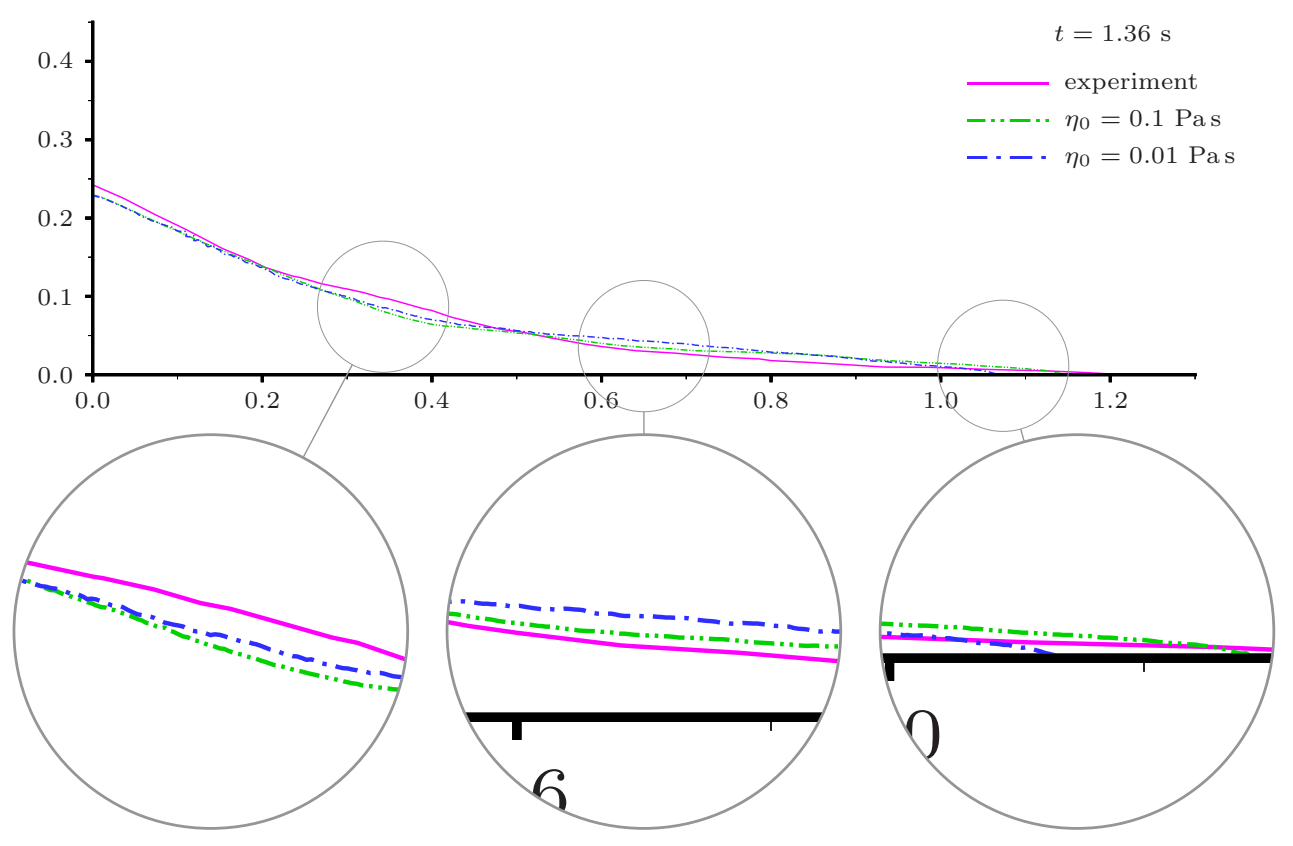

Figure 20: Profiles of the granular mass at the final time of the mass spreading obtained with the DP-rheology for the $(0,0.2) \times(0,0.4)$ initial column, where unit of length is metre. Profiles obtained with $\eta_{0}=10^{-1} \mathrm{~Pa} \mathrm{~s}$ and $\eta_{0}=10^{-2} \mathrm{Pas}$ are shown and compared with the experimental result. The mesh resolution is $h=1.796875 \times 10^{-3} \mathrm{~m}$ and the basal friction angle is $15^{\circ}$.

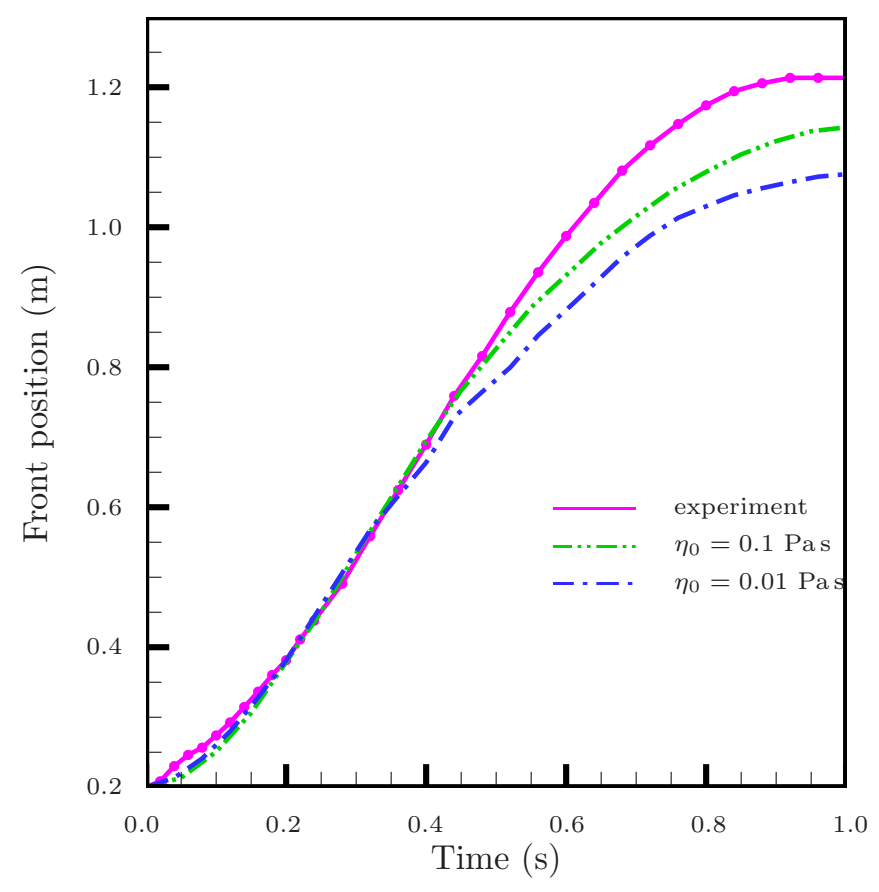

Figure 21: Time evolution of the front position during the spreading of the granular mass. The front stops at $t=0.9 \mathrm{~s}$ and the upper part of the basal deposit grows up to $t=1.36 \mathrm{~s}$ where a steady state is reached. Results obtained with $\eta_{0}=10^{-1} \mathrm{~Pa} \mathrm{~s}$ and $\eta_{0}=10^{-2} \mathrm{~Pa}$ s are shown and compared with the experimental result. The mesh resolution is $h=1.796875 \times 10^{-3} \mathrm{~m}$ and the basal friction angle is $15^{\circ}$. 


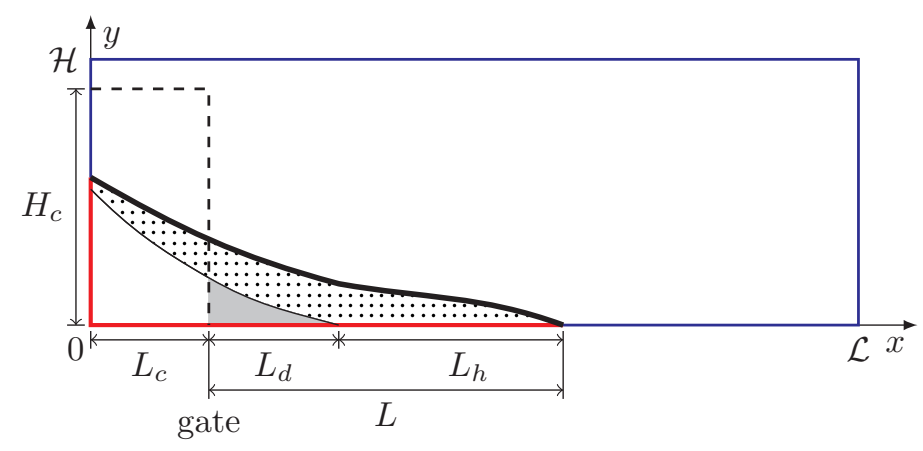

Figure 22: Granular flow structure determined by the upper flow surface (bold black) and the interface with the basal deposit (thin black). The wall contact surface between the granular flow and the channel is shown in red. The dashed line represents the initial granular column. The length of the granular mass on the $x$-axis, measured from the gate, $L$ is decomposed into the basal deposit length $L_{d}$ and the length of the sliding head $L_{h}$.

induces a larger characteristic velocity and so, for a similar resolution, the time step has to be decreased. For the friction angle, we chose the value $15^{\circ}$, as stated above.

The granular mass profile at steady state $(t=1.36 \mathrm{~s})$, shown on Figure 20, is weakly influenced by the value of the constant viscosity and is close to the experimental one. Both simulations predict the same height at the back wall and have profiles almost indistinguishable in the range $x \in[0,0.4] \mathrm{m}$. On the interval $x \in[0.6,0.8] \mathrm{m}$, a slightly thicker granular deposit is obtained with $\eta_{0}=10^{-2} \mathrm{~Pa}$ s. This results in a smaller runout distance: the front stops at $1.08 \mathrm{~m}$ compared with $1.145 \mathrm{~m}$ when $\eta_{0}$ is set to $10^{-1} \mathrm{~Pa}$ s. Time propagations of the front during the collapse of the granular column, represented on figure 21, are similar for both values of $\eta_{0}$ : the phase of propagation at constant velocity lasts longer in the experiment. Deceleration occurs in the numerical simulations near $t=0.6 \mathrm{~s}$ compared with $0.75 \mathrm{~s}$ in the experiment.

Therefore, as in section 4.1.4, we found that the numerical results are weakly dependent on the value of the constant viscosity: the main features of the flow dynamics and the profile of the granular mass after its spreading are recovered. This indicates that the collapse of a dense and dry granular column seems to be mainly governed by the Drucker-Prager plasticity criterion.

\subsubsection{Structure of the granular flow and characteristics of the deposit}

From measurements and analyzes of experiments, it has been shown in [20] and $[3$ that the flow of a granular material during the collapse of a column consists of a basal deposit growing at a nearly constant rate and overlain by a moving layer with a sliding head where most of the flow motion occurs. Following $[3]$, we aim to characterize both the basal deposit and the flow head by computing several related quantities and by comparing numerical and experimental results. To the best of our knowledge, we present the first comparison between simulations and experiments accounting for the internal dynamics of dambreak granular flows.

On figure 22 the granular mass, at any time during its spreading, is schematically split into a basal deposit extending from the back wall of the channel up to the distance $L_{d}+L_{c}$ where $L_{d}$ is the length of the deposit measured from the gate, which is located at $x=L_{c}$ from the origin. The flow occurs in the layer above the basal deposit and below the interface with ambient air: the dotted area on figure 22. The flowing layer can be characterized by its heigth, hereafter denoted by $h_{\ell}$, which is defined as the difference between the heigth of the whole granular mass and the heigth of the basal deposit. On figure 24, the heigth of the flowing layer is plotted as a function of the distance from the back wall at the same times as on figure 22. Note that for times $t \leq 0.72 \mathrm{~s}$, which correspond to the acceleration and constant velocity phases, $h_{\ell}$ grows almost linearly with $x$ until a peak is reached near $x=L_{c}+L_{d}$, which is the location of the front of the basal deposit. The peak location moves right with time from $x=20 \mathrm{~cm}$, which is the location of the gate, up to $x=45 \mathrm{~cm}$. For $x \geq L_{c}+L_{d}, h_{\ell}$ decays almost linearly until it vanishes at $x=L_{c}+L$, which is the location of the front of the granular mass. The time derivatives of $h_{\ell}$ (see figure (25)) also clearly exhibit two distinct regions : $h_{\ell}$ decreases with time from the location of the back wall up to that of the peak, observed on figure (24), followed by an increase of $h_{\ell}$ as function of time.

The foremost part of the flowing layer sliding on the bottom wall is named the head of the granular flow and is characterized by the length $L_{h}$, which is defined by: $L_{h}=L-L_{d}$ where $L$ is the total length of the granular mass measured from the gate. The basal deposit can also be characterized by the area $A_{d}$ (gray 

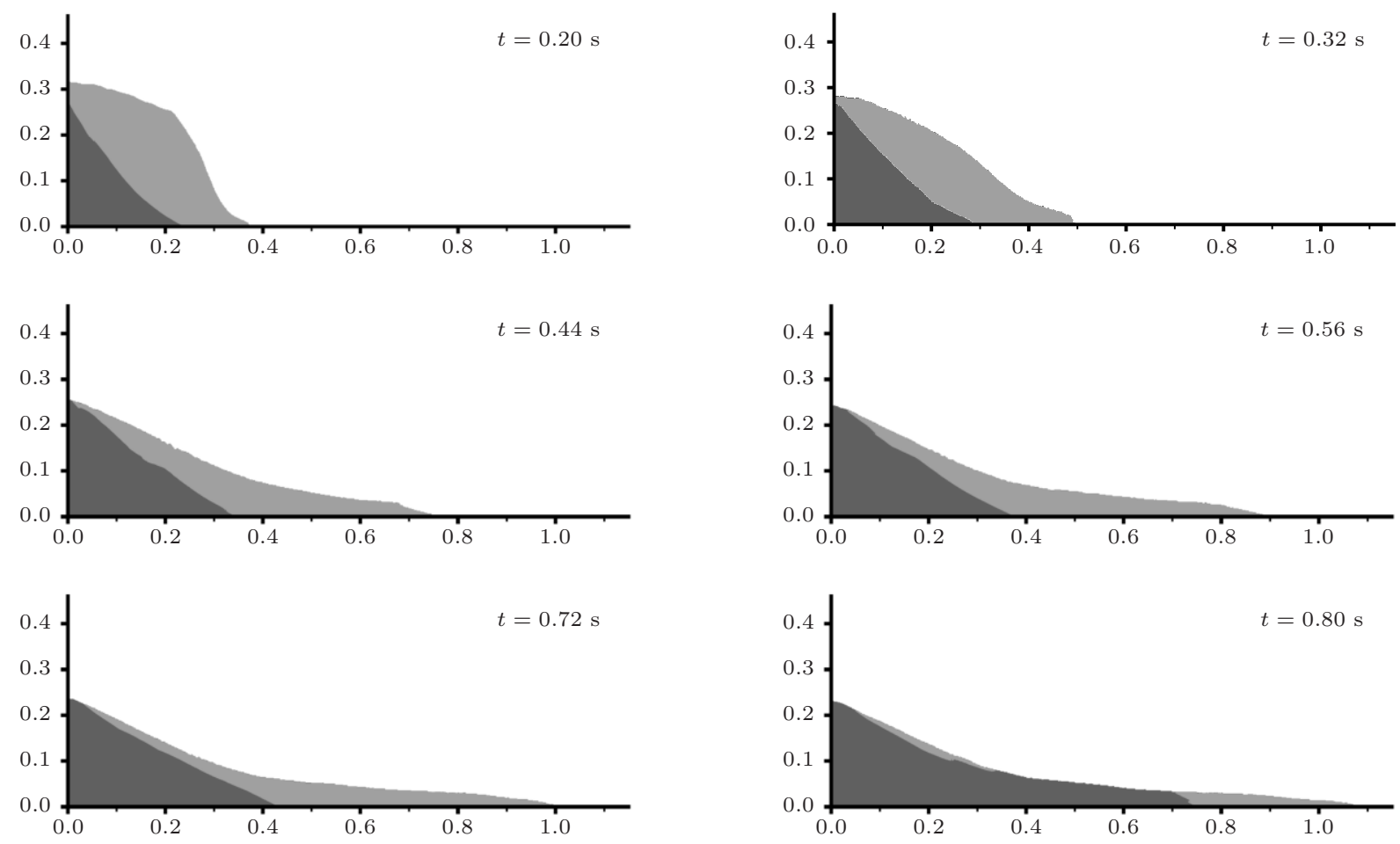

Figure 23: Basal deposit (dark gray) and flowing layer (light gray) of the granular mass at different times obtained with the DPrheology and with a threshold equals to 7.5 percent of the maximum velocity. The numerical resolution is $h=1.796875 \times 10^{-3}$ $\mathrm{m}(n=256)$, the computational domain is $\Omega=(0,1.38) \times(0,0.46)$, where unit of length is metre, the basal friction angle is $\alpha_{\mathrm{b}}=15^{\circ}$ and the constant viscosity is $\eta_{0}=0.1 \mathrm{~Pa}$.

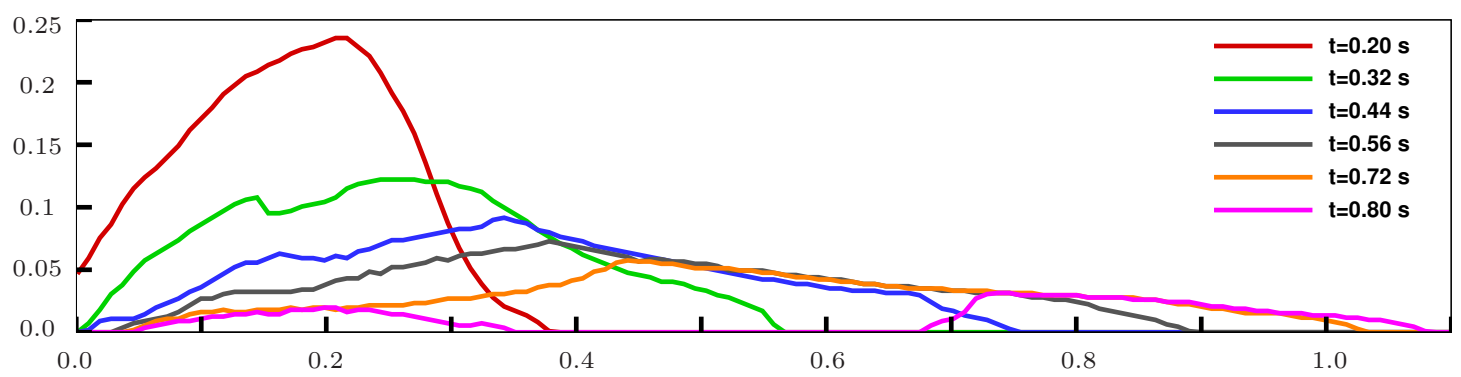

Figure 24: Heigth of the flowing layer (dotted area on Figure 22 at times $t=0.2 \mathrm{~s}, 0.32 \mathrm{~s}, 0.44 \mathrm{~s}, 0.56 \mathrm{~s}, 0.72 \mathrm{~s}$ and $0.8 \mathrm{~s}$. The numerical resolution is $h=1.796875 \times 10^{-3} \mathrm{~m}(n=256)$, the computational domain is $\Omega=(0,1.38) \times(0,0.46)$, where unit of length is metre, the basal friction angle is $\alpha_{\mathrm{b}}=15^{\circ}$ and the constant viscosity is $\eta_{0}=0.1 \mathrm{~Pa}$.

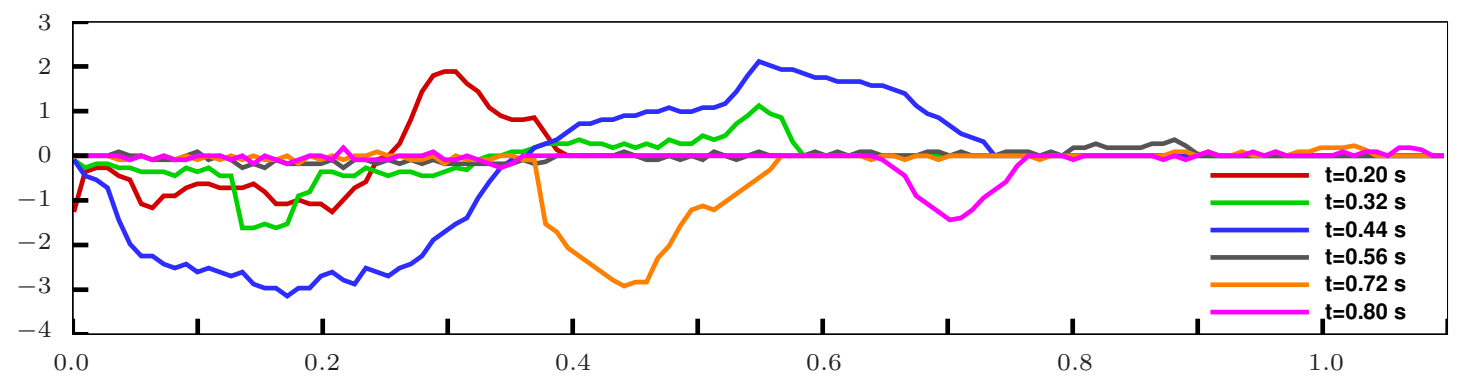

Figure 25: Time derivative of the heigth of the flowing layer (dotted area on Figure 22 at times $t=0.2 \mathrm{~s}, 0.32 \mathrm{~s}, 0.44$ $\mathrm{s}, 0.56 \mathrm{~s}, 0.72 \mathrm{~s}$ and $0.8 \mathrm{~s}$. The numerical resolution is $h=1.796875 \times 10^{-3} \mathrm{~m}(n=256)$, the computational domain is $\Omega=(0,1.38) \times(0,0.46)$, where unit of length is metre, the basal friction angle is $\alpha_{\mathrm{b}}=15^{\circ}$ and the constant viscosity is $\eta_{0}=0.1$ $\mathrm{Pa}$ s. 

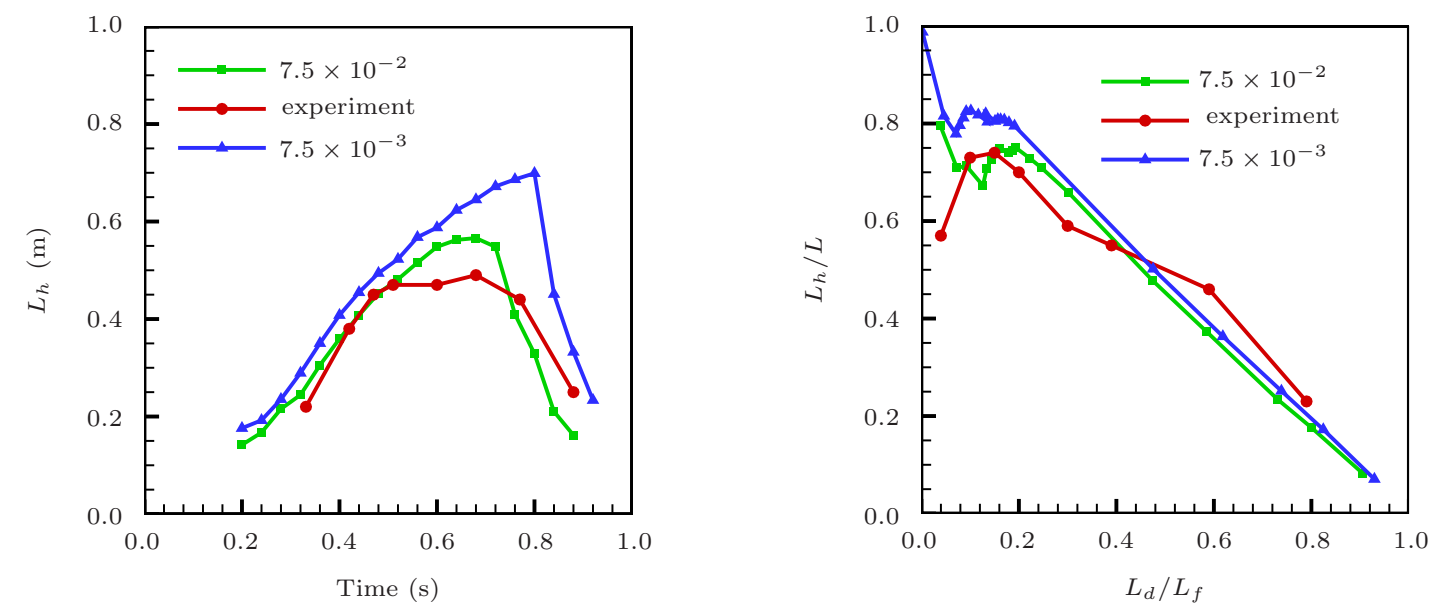

Figure 26: Structure of the granular flow for $H_{c} / L_{c}=2$. Time evolution of the length of the sliding head $L_{h}$ (left). $L_{h} / L$ represented as a function of the ratio $L_{d} / L_{f}$ (right) where $L_{f}$ is the runout distance. Results, obtained with the basal coefficient $\tan \left(15^{\circ}\right)$ and with the constant viscosity $\eta_{0}=0.1 \mathrm{~Pa} \mathrm{~s}$, are compared with values measured from the experiment.
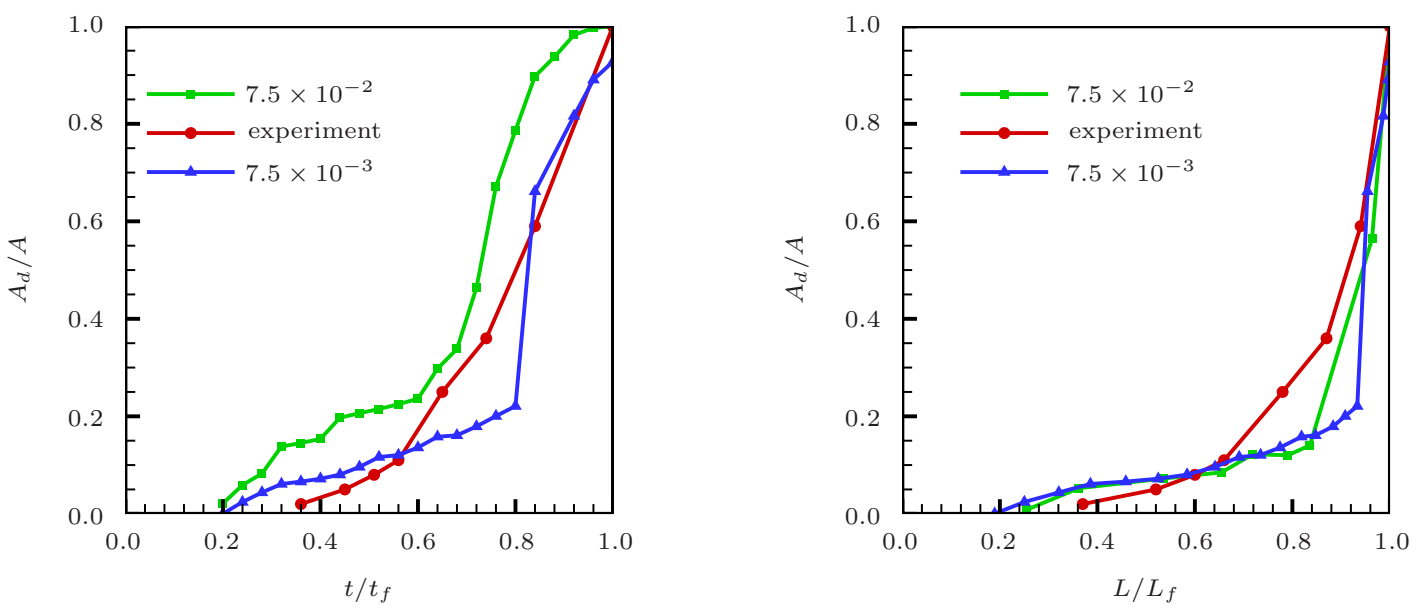

Figure 27: Structure of the granular flow for $H_{c} / L_{c}=2$. Deposit area $A_{d}$ (gray region on figure 22 normalized by the total area $A$ of the granular flow outside the reservoir as function of normalized time (left) and position (right). The final time $t_{f}$ is the the time where the granular flow reaches the runout distance $L_{f}$. Results obtained with basal coefficients $\tan \left(15^{\circ}\right)$ and $\eta_{0}=0.1 \mathrm{~Pa}$ s are compared with values measured from the experiment. 


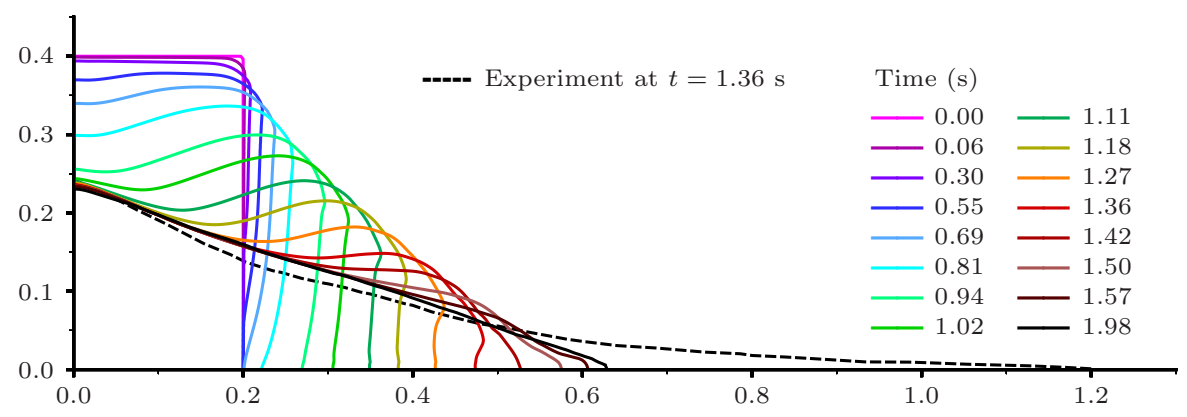

Figure 28: Profiles of the granular mass at different times, predicted by the HP-model. The numerical resolution is $h=$ $3.90625 \times 10^{-3} \mathrm{~m}(n=128)$, the computational domain is $\Omega=(0,4) \times(0,0.5)$, here unit of length is metre, and the basal friction angle is $\alpha_{\mathrm{b}}=19^{\circ}$.

area on figure 22, which is equal to the area of the deposit in the channel.

Due to short wave oscillations, the strain-rate can not be used to define the interface between the basal deposit and the overlaying moving layer because bands of large and small gradients alternate (see figure 19p. Notice that these oscillations characterize also the pressure (see figure 18). By contrast, the velocity norm (see figure 17) appears to be smooth. Therefore, we used the norm of the velocity field as a discriminant quantity and we defined the basal deposit as the region inside the granular mass where the velocity norm was below a given threshold. By using a cut-off value 7.5 percent of the maximum velocity norm, the deposit and the moving layer are clearly obtained at different times during the collapse of the granular mass as shown on figure 23. A similar decomposition of the granular flow can also be recovered for a ten times smaller value of the threshold. This is no longer the case for smaller values due to the resolution of the numerical simulation. In this regard, tests showed that the cut-off value decreased with the mesh size and the time step. In order to compare with the experiment, we quantify the basal deposit by computing the lengths $L_{h}, L_{d}$ as well as the area $A_{d}$ with the two aforementioned cut-off values, namely 7.5 and 0.75 percent of the maximum velocity norm.

As observed in the experiment, the length $L_{h}$ of the sliding head increases linearly as the time evolves, then reaches a maximum value and finally decreases. As in the experminent, a plateau is obtained in the numerical simulation when the largest value of the threshold is used. In the latter, $L_{h}$ reaches a larger maximum value ( $0.56 \mathrm{~m}$ in the simulation compared to $0.49 \mathrm{~m}$ in the experiment) and the plateau is narrower. Finally, a similar decrease is obtained for $t \geq 0.8 \mathrm{~s}$ (see figure 26. left curve). Until the final time of the collapse, the granular mass reaches the runout distance $L_{f}$ (see figure 16) while the basal deposit progressively represents the entire granular mass. Also shown on figure 26 (right curve) is the ratio $L_{h} / L$, which decreases linearly with the distance $L_{d} / L_{f}$. The numerical simulation matches the experimental data and shows a perfect linear dependency. Note that the curves obtained for both cut-off values have the same linear decay.

The fraction of the basal deposit area $A_{d}$ over the area $A$ of the whole granular material in the channel slowly increases during the stage of constant speed propagation, that is for $t \in\left(t_{1}, t_{2}\right)$, reaching approximately $20-25 \%$ (see figures 27). During the last stages, $A_{d} / A$ increases more rapidly until motion in the granular mass stops, so that $A_{d}=A$. Note that the experimental and numerical curves have the same trends but the numerical values are obtained at a slightly earlier time. However, a better agreement between the simulation and the experiment is obtained when the ratio $A_{d} / A$ is plotted against the normalized deposit length $L_{d} / L_{f}$. Again, the curves corresponding to the two different values of the threshold are close to each other.

As a summary, by analyzing the internal structure of the granular flow simulated with the DP-rheology, a good agreement with the experiment is obtained. Again, to the best of our knowledge, such study is presented here for the first time.

\subsubsection{The HP-rheology}

As for the column with aspect ratio of 0.7 (section 4.1.5), the HP-model fails to reproduce both the flow dynamics and the final deposit for the taller column (see figure 28). The runout distance is underestimated by nearly $50 \%$ though the height on the back wall is correctly predicted. As already mentioned, the strength of the yield stress defined as proportional to the hydrostatic pressure is stratified and far too strong, thus inhibiting motion of the right basal part of the column, which destabilizes in a non-physical manner from above. The simulation reaches a steady state at $t=1.98 \mathrm{~s}$ while the final time is found to be $t_{f}=1.36$ 

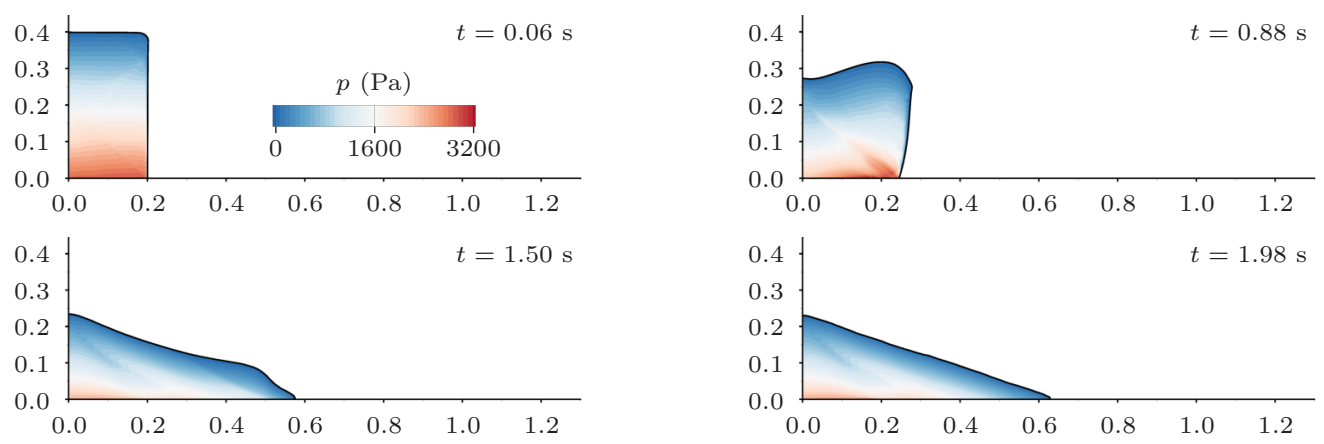

Figure 29: Pressure $p$ (in Pa) at different times during the granular mass slump simulated with the HP-model. The numerical resolution is $h=3.90625 \times 10^{-3} \mathrm{~m}(n=128)$, the computational domain is $\Omega=(0,4) \times(0,0.5)$, where unit of length is metre, and the basal friction angle is $\alpha_{\mathrm{b}}=19^{\circ}$.

$\mathrm{s}$ in the experiment. As expected and due to the well-posedness of the HP-model, the computed dynamic pressure has no visible small-scale oscillations, in contrast to the DP-model (see figure 29).

\section{Summary and conclusions}

Numerical simulations of the collapse of dry columns of glass beads over an horizontal plane have been performed with a two-phase version of a bi-projection scheme. In this study, comparisons with experiments and other (published) numerical results are reported. Two experiments of the collapse of columns of glass beads, with diameters respectively equal to $0.7 \mathrm{~mm}$ and $0.08 \mathrm{~mm}$, and aspect ratios respectively equal to 0.7 and 2, were considered. For the latter, numerical simulations are presented and analyzed for the first time. In that case, our simulations are likely to be more accurate than depth-averaged models as the aspect ratio exceeds the unity.

The granular medium is modeled as a continuum with a viscoplastic rheology, derived from the $\mu(I)$ rheology. The plasticity criterion takes the form of a Drucker-Prager criterion with a yield which is proportional to the pressure and a friction coefficient. The viscosity varies in space and time, depending on the norm of the strain-rate tensor and the pressure. In order to reduce the computational cost, the spatio-temporal variable viscosity of the $\mu(I)$-rheology is replaced by a constant viscosity. The influence of this value on the results has been studied by decreasing the viscosity so that it becomes negligible compared to the effective viscosity induced by the Drucker-Prager criterion. The dependency of the numerical results upon the value of the constant viscosity was found to be very weak, indicating that the flow dynamics during the collapse of a dense and dry granular column is essentially governed by the Drucker-Prager plasticity criterion.

Rewriting the $\mu(I)$-rheology in the framework of viscoplastic models is convenient and allows to apply specific algorithms developed in order to handle the non-differentiable definition of the plastic part of the stress tensor. Based on previous works, the non-differentiability of the yield stress rheology is handled with a projection formulation allowing a computation of the plastic part of the stress tensor through a fixed point procedure having a geometric convergence. A level-set formulation for the momentum and mass conservation equations is used. The interface between the granular material and the ambient air is tracked with highorder schemes and with an appropriate treatment near boundaries. Coulomb friction boundary conditions are applied on the walls so that sliding of the front during the spreading of the granular mass is allowed. Using the level-set method for the collapse of a granular column is challenging as this method is known to have difficulties to reproduce accurately the sliding of a front on a wall. In the present study, however, the level-set method coupled with Coulomb friction conditions on the horizontal wall is able to capture a very thin front of the granular mass, almost as thin as the experimental one.

Incompressible models with a Drucker-Prager plasticity criterion are known to be ill-posed, in the sense that small-scale oscillations may appear and grow, leading to instabilities. Despite this ill-posedness, the DP-model provides accurate predictions of the dynamics, the runout distance and the shape of the granular mass during the collapse for both experiments. In the case with aspect ratio 0.7 , the mesh size was decreased down to the diameter of the glass beads $(0.7 \mathrm{~mm})$ and a remarkably accurate prediction of the interface between the granular flow and the ambient air is recovered. It appears that, for this type of granular flows, simulations remain stable despite oscillations characterized by thin bands of plug zones separated by thin bands of high gradient, and are not affected when the viscosity is decreased by two orders of magnitude, down 
to $10^{-3} \mathrm{~Pa}$ s. In the area close to the vertical back wall, the numerical results perfectly fit the experimental ones. The four different stages characterizing granular mass spreading and observed in experiments are well predicted by the DP-model. Also, the granular mass during its collapse is known to separate into a basal deposit over which flows a thin layer of materials with a sliding head. For the taller initial column, the internal dynamics of the granular mass, namely the static-mobile interface between the basal deposit and the flowing layer, is well captured and a good agreement with the experiment is found. We present for the first time numerical quantification of the static and moving parts of a granular material during its spreading.

Another viscoplastic model, with constant viscosity but different threshold is also proposed and studied. The dynamic pressure of the flow in the definition of the yield in the Drucker-Prager rheology is replaced by the hydrostatic pressure (HP) derived from the flow height. The HP-model is known to be well-posed but it fails to reproduce the dynamics of the collapse of dry granular columns. Nevertheless, the results are reported as they can provide a stiff numerical test case for viscoplastic dambreaks. Indeed, the interface rolls up before the head of the granular mass touches the bottom wall. Intermediate profiles are not physically relevant because the vertically stratified yield stress is too strong and inhibits sliding of the front, so that the granular column destabilizes from the top. Surprisingly, an acceptable final deposit is predicted for an initial column with aspect ratio 0.7 , although the runout distance is underestimated, but this is no longer the case when the aspect ratio exceeds the unity.

In order to develop further the present work, columns with larger aspect ratios could be considered and simulations with finer resolutions could be investigated in the case of beads smaller than $0.08 \mathrm{~mm}$. We plan also to extend our code to three-dimensional geometries, possibly with lateral walls. Interesting test cases are the collapse of 3D columns and fast flows on inclined planes for which lateral walls may have non negligible effects (see [51]).

This work is supported by the French Government Laboratory of Excellence initiative $\mathrm{n}^{\circ}$ ANR-10-LABX0006, by the French National Research Agency (ANR) RAVEX project, and by the French National Joint Research Program TelluS of INSU and INSMI CNRS (National Centre for Scientific Research). This is Laboratory of Excellence ClerVolc contribution number 402. The numerical simulations have been performed on a DELL cluster with 32 processors Xeon E2650v2 (8 cores), 1 To of total memory and an infiniband (FDR $56 \mathrm{~Gb} / \mathrm{s})$ connecting network.

Declaration of Interests. The authors report no conflict of interest.

\section{References}

[1] GDR MiDi, On dense granular flows, Eur. Phys. J. E14 (2004) 341-365. doi:doi:310.1140/epje/ i2004-10025-10021.

[2] R. Delannay, A. Valance, A. Mangeney, O. Roche, P. Richard, Granular and particle-laden flows: from laboratory experiments to field observations, J. Phys. D Appl. Phys. 50 (5) (2017) 053001. doi: 10.1088/1361-6463/50/5/053001.

[3] O. Roche, Depositional processes and gas pore pressure in pyroclastic flows: an experimental perspective, B. Volcanol. 74 (8) (2012) 1807-1820.

[4] A. Mangeney-Castelnau, F. Bouchut, J. P. Vilotte, E. Lajeunesse, A. Aubertin, M. Pirulli, On the use of saint venant equations to simulate the spreading of a granular mass, J. Geophys. Res. Solid Earth 110 (B9) (2005). doi:10.1029/2004JB003161.

[5] R. R. Kerswell, Dam break with coulomb friction: A model for granular slumping?, Phys. Fluids 17 (5) (2005) 057101. doi:10.1063/1.1870592.

[6] E. Larrieu, L. Staron, E. Hinch, Raining into shallow water as a description of the collapse of a column of grains, J. Fluid Mech. 554 (2006) 259-270. doi:10.1017/S0022112005007974.

[7] V. Gueugneau, K. Kelfoun, O. Roche, L. Chupin, Effects of pore pressure in pyroclastic flows: Numerical simulation and experimental validation, Geophys. Res. Lett. 44 (5) (2017) 2194-2202. doi:10.1002/ 2017GL072591.

[8] L. Lacaze, J. Phillips, R. Kerswell, Planar collapse of a granular column: Experiments and discrete element simulations, Phys. Fluids 20 (6) (2008) 063302. doi:10.1063/1.2929375 
[9] L. Girolami, V. Hergault, G. Vinay, A. Wachs, A three-dimensional discrete-grain model for the simulation of dam-break rectangular collapses: comparison between numerical results and experiments, Granul. Matter 14 (3) (2012) 381-392. doi:10.1007/s10035-012-0342-3. URL https://doi .org/10.1007/s10035-012-0342-3

[10] G. B. Crosta, S. Imposimato, D. Roddeman, Numerical modeling of 2-d granular step collapse on erodible and nonerodible surface, J. Geophys. Res. Earth Surface 114 (F3) (2009). doi:10.1029/ 2008JF001186

[11] Y. Jop, P.and Forterre, O. Pouliquen, A constitutive law for dense granular flows, Nature 441 (2006) $727-730$.

[12] P. Lagrée, L. Staron, S. Popinet, The granular column collapse as a continuum: Validity of a twodimensional Navier-Stokes model with a $\mu(I)$-rheology, J. Fluid Mech. 686 (2011) 378-408.

[13] I. Ionescu, A. Mangeney, F. Bouchut, O. Roche, Viscoplastic modeling of granular column collapse with pressure-dependent rheology, J. Non-Newt. Fluid 219 (2015) 1-18.

[14] N. Martin, I. R. Ionescu, A. Mangeney, F. Bouchut, M. Farin, Continuum viscoplastic simulation of a granular column collapse on large slopes: $\mu(I)$ rheology and lateral wall effects, Phys. Fluids 29 (1) (2017) 013301. doi:10.1063/1.4971320.

[15] J. Gray, A. Edwards, A depth-averaged $\mu(i)$-rheology for shallow granular free-surface flows, Journal of Fluid Mechanics 755 (2014) 503-534. doi:10.1017/jfm.2014.450.

[16] N. J. Balmforth, R. R. Kerswell, Granular collapse in two dimensions, Journal of Fluid Mechanics 538 (2005) 399-428. doi:10.1017/S0022112005005537

[17] A. Mangeney, O. Roche, O. Hungr, N. Mangold, G. Faccanoni, A. Lucas, Erosion and mobility in granular collapse over sloping beds, J. Geophys. Res. Earth Surface 115 (F3) (2010). doi:10.1029/ 2009JF001462

[18] D. Schaeffer, Instability in the evolution equations describing incompressible granular flow, J. Differ. Equations 66 (1) (1987) 19-50.

[19] D. Schaeffer, E. Pitman, Ill-posedness in three-dimensional plastic flow, Commun. Pure Appl. Math. 41 (7) (1988) 879-890.

[20] O. Roche, S. Montserrat, Y. Niño, A. Tamburrino, Pore fluid pressure and internal kinematics of gravitational laboratory air-particle flows: Insights into the emplacement dynamics of pyroclastic flows, J. Geophys. Res. Solid Earth 115 (B9) (2010) B09206. doi:10.1029/2009JB007133.

[21] L. Chupin, J. Mathé, Existence theorem for homogeneous incompressible Navier-Stokes equation with variable rheology, Eur. J. Mech. B-Fluid 61 (2017) 135-143. doi:https://doi.org/10.1016/ j.euromechflu.2016.09.020.

[22] D. Schaeffer, T. Barker, D. Tsuji, P. Gremaud, M. Shearer, J. Gray, Constitutive relations for compressible granular flow in the inertial regime, J. Fluid Mech. 874 (2019) 926-951.

[23] L. Chupin, T. Dubois, A bi-projection method for Bingham type flows, Comput. Math. Appl. 72 (5) (2016) 1263-1286. doi:10.1016/j.camwa.2016.06.026 URL http://dx.doi.org/10.1016/j.camwa.2016.06.026

[24] R. Chalayer, L. Chupin, T. Dubois, A bi-projection method for incompressible bingham flows with variable density, viscosity, and yield stress, SIAM J. Numer. Anal. 56 (4) (2018) 2461-2483. arXiv: https://doi.org/10.1137/17M113993X, doi:10.1137/17M113993X. URL https://doi.org/10.1137/17M113993X

[25] R. Glowinsky, J.-L. Lions, R. Trémolières, Numerical Analysis of Variational Inequalities, NorthHolland, Amsterdam, 1981.

[26] R. Glowinsky, P. Le Tallec, Augmented Lagrangian and Operator-Splitting Methods in Nonlinear Mechanics, Studies in Applied and Numerical Mathematics, 1989. 
[27] L. Muravleva, Uzawa-like methods for numerical modeling of unsteady viscoplastic Bingham medium flows, Appl. Numer. Math. 93 (2015) 140-149. doi:10.1016/j.apnum.2014.06.001.

URL http://dx.doi.org/10.1016/j.apnum.2014.06.001

[28] J. Guermond, P. Minev, J. Shen, An overview of projection methods for incompressible flows, Comput. Methods Appl. Mech. Eng. 195 (44) (2006) 6011 - 6045. doi:https://doi.org/10.1016/j.cma.2005. 10.010.

[29] M. Sussman, P. Smereka, S. Osher, A level set approach for computing solutions to incompressible two-phase flow, J. Comput. Phys. 114 (1994).

[30] G. Della Rocca, G. Blanquart, Level set reinitialization at a contact line, J. Comput. Phys. 265 (2014) $34-49$.

[31] Y. Liu, N. Balmforth, S. Hormozi, D. Hewitt, Two-dimensional viscoplastic dambreaks, J. Non-Newt. Fluid 238 (2016) 65 - 79. doi:https://doi.org/10.1016/j.jnnfm.2016.05.008.

[32] B. Andreotti, Y. Forterre, O. Pouliquen, Les Milieux Granulaires, Entre fluide et Solide, Savoirs Actuels, EDP sciences, 2011.

[33] S. Savage, The mechanics of rapid granular flows, Adv. Appl. Mech. 24 (1984) 289-366.

[34] C. Ancey, P. Coussot, P. Evesque, A theoretical framework for granular suspensions in a steady simple shear flow, J. Rheol. 43 (1999) 1673-1699.

[35] S. Osher, R. Fedkiw, Level Set Methods and Dynamic Implicit Surfaces, Vol. 153 of Applied Mathematical Sciences, Springer-Verlag, New-York, 2003.

[36] M. Sussman, K. Smith, M. Hussaini, M. Otha, R. Zhi-Wei, A sharp interface method for incompressible two-phase flows, J. Comput. Phys. 221 (2) (2007).

[37] Y. Chang, T. Hou, B. Merriman, S. Osher, A level set formulation of eulerian interface capturing methods for incompressible fluid flows, J. Comput. Phys. 124 (1996).

[38] M. Sussman, E. Fatemi, P. Smereka, S. Osher, An improved level set method for incompressible twophase flows, Comp. Fluids 27 (5-6) (1998).

[39] C.-W. Shu, S. Osher, Efficient implementation of essentially non-oscillatory shock-capturing schemes, J. Comput. Phys. 77 (2) (1988) 439-471.

[40] G.-S. Jiang, D. Peng, Weighted eno schemes for hamilton-jacobi equations, SIAM J. Sci. Comput. 21 (6) (2000).

[41] S. Gottlieb, C.-W. Shu, Total variation diminishing Runge-Kutta schemes, Math. Comp. 67 (221) (1998) $73-85$.

[42] C. Min, On reinitializing level set functions, J. Comput. Phys. 229 (2010).

[43] A. Chorin, Numerical solution of the Navier-Stokes equations, Math. Comp. 22 (1968) 745-762. doi: $10.2307 / 2004575$. URL http://dx.doi.org/10.2307/2004575

[44] R. Temam, Sur l'approximation de la solution des équations de Navier-Stokes par la méthode des pas fractionnaires. II, Arch. Rational Mech. Anal. 33 (1969) 377-385. doi:10.1007/BF00247696. URL http://dx.doi.org/10.1007/BF00247696

[45] F. Harlow, J. Welch, Numerical calculation of time-dependent viscous incompressible flow of fluid with free surface, Phys. Fluids 12 (8) (1965) 2182-2189.

[46] S. Balay, S. Abhyankar, M. Adams, J. Brown, P. Brune, K. Buschelman, L. Dalcin, A. Dener, V. Eijkhout, W. Gropp, D. Kaushik, M. Knepley, D. A. May, L. C. McInnes, R. Tran Mills, T. Munson, K. Rupp, P. Sanan, B. Smith, S. Zampini, H. Zhang, H. Zhang, PETSc users manual, Tech. Rep. ANL95/11 - Revision 3.10, Argonne National Laboratory (2018).

URL http://www.mcs.anl.gov/petsc 
[47] S. Balay, S. Abhyankar, M. Adams, J. Brown, P. Brune, K. Buschelman, L. Dalcin, A. Dener, V. Eijkhout, W. Gropp, D. Kaushik, M. Knepley, D. A. May, L. C. McInnes, R. Tran Mills, T. Munson, K. Rupp, P. Sanan, B. Smith, S. Zampini, H. Zhang, H. Zhang, PETSc Web page, http://www.mcs.anl.gov/petsc (2018).

URL http://www.mcs .anl.gov/petsc

[48] L. Lacaze, R. Kerswell, Axisymmetric granular collapse: A transient 3d flow test of viscoplasticity, Phys. Rev. Lett. 102 (2009) 108305. doi:10.1103/PhysRevLett.102.108305

[49] T. Barker, D. Schaeffer, P. Bohórquez, J. Gray, Well-posed and ill-posed behaviour of the $\mu(I)$-rheology for granular flow, J. Fluid Mech. 779 (2015) 794-818.

[50] T. Barker, J. Gray, Partial regularisation of the incompressible $\mu(I)$-rheology for granular flow, J. Fluid Mech. 828 (2017) 5-32.

[51] N. Brodu, R. Delannay, A. Valance, R. P., New patterns in high-speed granular flows, J. Fluid Mech. 769 (2015) 218-228. doi:doi:210.1017/jfm.2015.1109. 